Research Article

iMedPub Journals

www.imedpub.com

\section{Use of Different Types and Amounts of Dietary Fats to Redesign Pork}

\section{Abstract}

Title: Use of different types and amounts of dietary fats to redesign pork.

Background: Using high energy fat-supplemented diets in pork production can offer several economic advantages to producers when fat sources are cost effectively priced. Because of a decreased heat increment, dietary fat supplementation allows a greater proportion of dietary calories to be available for tissue synthesis when pigs are maintained at or above the thermo neutral zone. Previous research has indicated that diets that have increasing dietary saturated fatty acids (SFA) cause hypercholesterolemia, atherosclerosis development, and greater coronary heart disease risk in humans. When dietary polyunsaturated fatty acids (PUFA) are substituted for saturated fatty acids (SFA), decreased blood cholesterol concentration occurs. This information has caused the consumer to question the consumption of meat products, such as pork, which are perceived as being rich in SFAs. The objective of this study was to alter the polyunsaturated (PUFA) to saturated fatty acid (SFA) ratio in pork for better human nutrition.

Methods and findings: Pigs were fed either choice white grease or soybean oil at $10,20,30$, or $40 \%$ of total dietary calories. All diets were based on corn and soybean meal. The study used 54 pigs with six pigs per treatment. Initial and slaughter weights were 54 and $110 \mathrm{~kg}$, respectively. Skeletal muscle samples were taken from the longissimus dorsi, biceps femoris, and triceps brachii muscles. Adipose tissue samples were taken from the outer, middle, and inner $10^{\text {th }}$ rib backfat layers, perirenal adipose tissue, and an inter muscular adipose deposit within the ham. Total lipids were extracted; fatty acid methyl esters were formed by trans esterification and quantified by gas chromatography. Adding choice white grease or soybean oil to diets fed growing swine did not alter animal growth rates. The PUFA from the outer $10^{\text {th }}$ rib backfat layer showed linear increases $(P<0.05)$ when pigs were fed diets with increasingly greater soybean oil content, whereas the backfat from pigs fed diets containing greater choice white grease contents resulted in a linear increase $(P<0.05)$ of monounsaturated fatty acids (MUFA). Including soybean oil in the low-fat control diet at 30 and $40 \%$ increased the PUFA to SFA ratios $(\mathrm{P}: \mathrm{S})$ to 0.9 and $1.26(\mathrm{P}<0.05)$, respectively, in longissimus muscle. The MUFA content in the longissimus muscle was decreased by $30 \%(P<0.05)$ with the $40 \%$ soybean oil diet. Myristate, palmitate, and total SFA in longillssimus muscle decreased $27(P<0.05), 30(P<0.05)$, and 29\% $(P<0.05)$, respectively, with the $40 \%$ soybean oil diet.

Conclusions: Including choice white grease in the diet had minimal effects on the unsaturated to SFA muscular lipid ratios. In conclusion, high fat diets rich in unsaturated fatty acids can be used effectively to redesign pork for consumers wanting to decrease their consumption of saturated fatty acids.

Key words: Choice white grease; Soybean oil; Adipose tissues; Swine, Dietary fat

\section{Arlie D Penner, Murray L Kaplan, Lauren L Christian, Kenneth J Stalder* and Donald C Beitz}

Department of Animal Science, lowa State University, Ames, Iowa, USA

Corresponding author: Kenneth J Stalder

” stalder@iastate.edu

Department of Animal Science, lowa State University, Ames, lowa, USA.

Citation: Penner AD, Kaplan ML, Christian LL, Stalder KJ, Beitz DC (2018) Use of Different Types and Amounts of Dietary Fats to Redesign Pork. Crit Care Obst \& Gyne. Vol.2 No.1:3 


\section{Introduction}

Using high-energy fat-supplemented diets in pork production offers several economic advantages to producers. Because of a decreased heat increment, dietary fat supplementation allows a greater proportion of dietary calories to be available for tissue synthesis when pigs are maintained at or above the thermo neutral zone [1]. Previous research [2-4] suggested that increasing dietary saturated fatty acids (SFA) causes hypercholesterolemia, atherosclerosis development, and greater coronary heart disease risk in humans. Substituting dietary polyunsaturated fatty acids (PUFA) for saturated fatty acids (SFA) decreased blood cholesterol concentration $[5,6]$. This information has caused the consumer to question the consumption of meat products, such as pork, which are perceived as being rich in SFAs. Pork fat, however, is relatively rich in unsaturated fatty acids. Moreover, a recent meta-analysis of randomized control human trials demonstrated that total red meat (considered to be relatively rich in SFA) intake of $\geq 0.5$ servings per day does not negatively influence cardiovascular risk factors [7]. Recent research [8, 9] has caused one to question the severity of SFA on vascular health. Because much of the dietary fat consumed can be incorporated into body fat, fatty acid composition of porcine adipose tissue is influenced markedly by the fatty acid composition of dietary supplemental fats [10-12]. The fatty acid composition of pork may be strategic because some pork flavors results from heating carbonylcontaining compounds, and fatty acids are the principal source $[13,14]$. Therefore, decreasing carcass fat in pigs and decreasing the saturated to unsaturated fatty acid proportion would make pork more acceptable to health-conscious consumers. We hypothesized that increasing the unsaturated fatty acid content of the diet can increase the perceived healthfulness of the resulting pork products. The objective of this study was to determine the effects of adding choice white grease (CWG) or soybean oil (SBO) at $10,20,30$, or $40 \%$ of total calories to growing finishing swine diets on growth and performance, carcass characteristics and composition, and fatty acid composition of lipids of major adipose and skeletal muscle tissues.

\section{Materials and Methods}

The lowa State University Committee on Animal Care approved the procedures used for pork production at the lowa State University Swine Breeding Farm at the time this study was conducted.

\section{Animals}

Fifty-four crossbred barrows and gilts, approximately 19 weeks of age and $54 \mathrm{~kg}$ body weight, were assigned randomly into nine groups so that breed, gender, initial body weight, and litter origin were equalized among the groups of six pigs each. The crossbred makeup of the pigs was either $3 / 4$ Landrace and $1 / 4$ Hampshire or $3 / 4$ Hampshire and $1 / 4$ Landrace. Each group was assigned randomly to one of the nine diets described in Table 1. Pigs were fed the experimental diets until they weighed $110 \mathrm{~kg}$, which was for 10 weeks. Pigs were penned individually in $1.2 \times 1.7 \mathrm{~m}$ pens with

Table 1 Diet composition from a study evaluating feeding choice white grease (CWG) or soybean oil (SBO) to grow-finish pigs.

\begin{tabular}{|c|c|c|c|c|c|c|c|c|c|}
\hline \multirow[b]{2}{*}{ Item } & \multicolumn{9}{|c|}{ Diets $^{1}$} \\
\hline & Control & $10 \%$ CWG & $20 \%$ CWG & $30 \%$ CWG & $40 \%$ CWG & $10 \% \mathrm{SBO}$ & $20 \%$ SBO & $30 \% \mathrm{SBO}$ & $40 \% \mathrm{SBO}$ \\
\hline \multicolumn{10}{|l|}{ Ingredients, $\%$ as fed } \\
\hline Ground shelled corn & 85.84 & 78.22 & 69.68 & 60.03 & 49.05 & 78.07 & 69.40 & 59.62 & 46.20 \\
\hline Soybean meal ${ }^{2}$ & 12.30 & 15.25 & 18.53 & 22.23 & 26.47 & 15.21 & 18.44 & 22.08 & 24.93 \\
\hline Choice white grease & - & 4.67 & 9.91 & 15.84 & 22.60 & - & - & - & - \\
\hline Solka Floc ${ }^{* 3}$ & - & - & - & - & - & - & - & - & 4.68 \\
\hline Soybean oil ${ }^{4}$ & - & - & - & - & - & 4.86 & 10.29 & 16.41 & 22.22 \\
\hline Calcium carbonate & 0.92 & 0.90 & 0.88 & 0.85 & 0.81 & 0.89 & 0.86 & 0.84 & 0.78 \\
\hline Dicalcium phosphate & 0.42 & 0.45 & 0.48 & 0.52 & 0.55 & 0.46 & 0.49 & 0.54 & 0.67 \\
\hline Salt & 0.27 & 0.26 & 0.27 & 0.28 & 0.27 & 0.26 & 0.27 & 0.27 & 0.28 \\
\hline TM and Vit. Mix & 0.25 & 0.25 & 0.25 & 0.25 & 0.25 & 0.25 & 0.25 & 0.25 & 0.25 \\
\hline \multicolumn{10}{|l|}{ Calculated nutrient values } \\
\hline $\mathrm{ME}^{6}(\mathrm{Mcal} / \mathrm{kg})$ & 3.35 & 3.55 & 3.77 & 4.01 & 4.29 & 3.54 & 3.75 & 3.98 & 4.04 \\
\hline Protein & 13.3 & 14.0 & 14.9 & 15.9 & 17.0 & 14.0 & 14.8 & 15.8 & 16.0 \\
\hline \multicolumn{10}{|l|}{ Analysis } \\
\hline Total lipids, \% & 2.19 & 5.86 & 10.31 & 15.76 & 19.64 & 6.31 & 10.79 & 15.62 & 22.59 \\
\hline DM, \% & 87.33 & 88.73 & 90.23 & 91.74 & 86.99 & 88.01 & 89.97 & 90.91 & 92.61 \\
\hline
\end{tabular}

${ }^{1} \mathrm{Abbr}$. $10 \%, 20 \%, 30 \%$, and $40 \%$ refer to amount of calories in diet from lipids, CWG = Choice white grease, SBO = Soybean oil.

${ }^{2} 48 \%$ crude protein.

${ }^{3}$ Fiber Sales and Development Corp, Urbana, $\mathrm{OH}$.

${ }^{4}$ Central Soya, Inc, Fort Wayne, IN, donated through the courtesy of D Strayer.

${ }^{5}$ Commercial trace mineral and vitamin mix contains (per kg): $33.4 \mathrm{~g}$ calcium, $109 \mathrm{~g}$ magnesium, $4 \mathrm{~g}$ copper, $410 \mathrm{mg}$ iodine, $32 \mathrm{~g}$ iron, $16 \mathrm{~g}$ manganese, $120 \mathrm{mg}$ selenium, $32 \mathrm{~g}$ zinc, $160 \mathrm{mg}$ cobalt, $2220 \mathrm{KIU}$ vitamin A, $709 \mathrm{KIU}$ vitamin $\mathrm{D}_{3}, 6.72 \mathrm{KIU}$ vitamin E, 883 mg vitamin K, $201 \mathrm{mg}$ menadione (synthetic vitamin K), 38,269 mg choline, 44,089 mg choline chloride, 14,108 mg niacin, $7067 \mathrm{mg}$ d-pantothenic acid, $1776 \mathrm{mg}$. riboflavin, and $11 \mathrm{mg}$ vitamin $B_{12}$. This premix was added at a rate of $2.5 \mathrm{~kg} / 1000 \mathrm{~kg}$ feed, as-fed basis.

${ }^{6}$ Metabolizable energy. 
concrete floors and an open flush gutter across the rear portion of the pen ( $30 \%$ of floor space) in an environmentally controlled building at approximately $18^{\circ} \mathrm{C}$. The building was located on the lowa State University Bilsland Swine Breeding Farm near Madrid, IA. Pigs were provided ad libitum access to water via automatic nipple drinkers and feed from individual feeders. Body weight and feed intake were recorded weekly.

\section{Diets}

Dietary ingredients and chemical composition are shown in Table 1. Diets were supplemented with either CWG or SBO at 10, 20, 30, or $40 \%$ of total calories. The fats utilized in this study were chosen because they represented two diverse fat sources commonly utilized and available in the Midwest United States where the majority of market pigs are fed. Further, each fat represented the spectrum of saturated fats fed to pigs with CWG being a commonly fed, highly-saturated fat source and SBO a source of unsaturated fat that could be fed to pigs. Fatty acid composition of CWG and SBO is given in Table 2. All diets were based on corn and soybean meal. Choice white grease has a melting point of about $32^{\circ} \mathrm{C}$ and was melted prior to adding to the diets to facilitate mixing. Solka Floc $^{\circledR}$ was added as an SBO absorbent. Diets were formulated to maintain a constant protein:energy ratio for all treatments and were prepared in batches several times during the experiment to maintain freshness. Samples from each diet were taken from all batches and stored at $-20^{\circ} \mathrm{C}$ until analysis. Gross energy from each experimental diet was verified by bomb calorimetry (Adiabatic Pan Oxygen Bomb Calorimeter, model 1241, Parr Instrument Co., Inc., Moline, IL). Protein content from each diet was determined by combustion [15]. Dicalcium phosphate, calcium carbonate, and a trace mineral and vitamin mixture were added to each diet in order to meet or exceed daily requirements for growing pigs [16]. There is no evidence in the literature that feeding these levels of fats the availability of other nutrients including protein that would impact the pig's health throughout the grow-finish production phase.

\section{Tissue sample collection}

At approximately $110 \mathrm{~kg}$ body weight, pigs were slaughtered at the federally inspected lowa State University Meat Laboratory after a 12-hour fast. Pigs were provided ad libitum water access during the fast. Pigs were stunned electrically and exsanguinated. After viscera removal, a perirenal adipose tissue sample was taken from each pig and stored at $-20^{\circ} \mathrm{C}$ until analysis. Samples were not taken from one carcass after slaughter, because it was condemned because tuberculosis lesions were found on it. Carcasses minus perirenal adipose tissue were weighed and then chilled at $-2^{\circ} \mathrm{C}$. At $24 \mathrm{~h}$ postmortem, backfat thickness and longissimus muscle area, color, marbling, and firmness were obtained by using standard procedures [17]. Carcasses were split longitudinally, and each half was weighed. The right half of each carcass was skinned mechanically and physically separated into skeletal muscle, bone, and adipose tissue. Skeletal muscle samples were taken from the longissimus muscle (10th rib), the biceps femoris muscle, and the triceps brachii muscle. Adipose tissue samples were collected from sites adjacent to the
Table 2 Fatty acid composition of choice white grease and soybean oil used in a grow-finish swine feeding study examining the effects of feeding fat on carcass quality and quantity.

\begin{tabular}{|c|c|c|}
\hline Item & $\mathrm{CWG}^{1}$ & $\mathrm{SBO}^{2}$ \\
\hline \multicolumn{3}{|l|}{ Fatty Acid ${ }^{3}$} \\
\hline $14: 0$ & 1.37 & 0.03 \\
\hline $15: 0$ & 0.07 & ND \\
\hline $16: 0$ & 26.1 & 10.4 \\
\hline $16: 1$ & 2.94 & 0.08 \\
\hline $17: 0$ & 0.38 & 0.07 \\
\hline $17: 1$ & 0.35 & 0.03 \\
\hline $18: 0$ & 11.0 & 4.2 \\
\hline $18: 1$ t n-9 & 1.34 & ND \\
\hline $18: 1 \mathrm{c} n-9$ & 44.1 & 22.4 \\
\hline $18: 2$ & 11.7 & 55.3 \\
\hline $18: 3 n-3$ & 0.22 & 7.30 \\
\hline $20: 0$ & $\mathrm{ND}^{4}$ & 0.16 \\
\hline $20: 1$ & 0.20 & ND \\
\hline $20: 2$ & 0.11 & ND \\
\hline SFA & 39.0 & 14.9 \\
\hline MUFA & 48.9 & 22.5 \\
\hline PUFA & 12.1 & 62.6 \\
\hline$P: S$ & 0.31 & 4.20 \\
\hline U:S & 1.56 & 5.71 \\
\hline
\end{tabular}

${ }^{1} \mathrm{CWG}=$ Choice white grease.

${ }^{2} \mathrm{SBO}=$ Soybean oil.

${ }^{3}$ Fatty acids are reported as a weight percentage of total fatty acids identified, SFA = total saturated fatty acids, MUFA = total monounsaturated fatty acids, PUFA = total polyunsaturated fatty acids, $\mathrm{P}: \mathrm{S}=$ ratio of total polyunsaturated to total saturated fatty acids, $\mathrm{U}: \mathrm{S}$ = ratio of total unsaturated (mono- and poly-) to total saturated fatty acids.

${ }^{4} \mathrm{ND}=$ not detected.

longissimus muscle (10th rib, inner, middle, and outer 10th rib backfat layers were separated at the visible connection tissue septum), adjacent to the biceps femoris muscle, adjacent to the triceps brachii muscle, adjacent to the navel area of the belly, and an internal intermuscular deposit within the ham. All samples were removed at 24 hours postmortem and stored at $-20^{\circ} \mathrm{C}$ until analysis.

\section{Chemical analyses}

All feed samples, skeletal muscles, and adipose tissues were lyophilized to obtain drymatter content. Total lipids were extracted from lyophilized samples in chloroform:methanol:water (1:2:0.8, $\mathrm{v}: \mathrm{v}: \mathrm{v})$ and dried under nitrogen [18]. Total lipid percentages in all samples were determined by gravimetric measurement. Fatty acid methyl esters (FAME) were prepared from wet tissue total lipid extracts by transesterification in methanol:benzene $(4: 1$, $\mathrm{v}: \mathrm{v})$ and acetyl chloride [19]. The hexane layer containing the FAME was pipetted into 400 ul vials and capped. Samples were injected into a Varian model 3400 gas chromatograph (Varian Analytical Instruments, Palo Alto, CA) with a Supelco 2380 column (Supelco, Inc., Bellefonte, PA) to separate FAME by retention times. Individual fatty acid identification and quantification were obtained by comparing eluted peak electronic integration with 
retention times from commercially available reference standards (Nuchek Prep., Elysian, MN).

\section{Statistical analyses}

Analyses of variance for a randomized complete block design were computed by using the GLM procedure [20]. Least squares means and the probability of differences between least squares means were computed. Preplanned orthogonal comparisons were used to determine differences among dietary treatments. These comparisons were linear effects by amount for each dietary lipid. A split-plot design was used to compare differences among individual tissues, anatomical locations, and tissue types.

\section{Results and Discussion}

\section{Growth and performance}

To evaluate dietary supplemental fat inclusion in grow-finish pig diets, several growth and performance measures were made (Table 3). Making these evaluations is needed to determine if similar or better pig performance can be expected so producers can estimate additional costs associated with feeding high fat grow - finish diets. Average daily feed intake (ADFI) decreased when CWG was added to grow-finish pig diets at 30 and $40 \%$ of energy. When SBO was added to the diet at 20,30 , and $40 \%$ of energy, ADFI decreased. Both CWG and SBO produced a linear $(P<0.001)$ response for ADFI. Average daily gain was similar for pigs from all treatments. A marked linear $(P<0.001)$ increase in feed efficiency (g BW growth / kg feed intake) was realized for pigs fed CWG or SBO. These results are consistent with results from a previous study [21] where pigs were a diet containing $5 \%$ animal fat, primarily from pork. Further, the results from Myer and Combs (1991) [22] who fed a diet with 3\% CWG to growingfinishing pigs, and Morgan et al. [23] who fed a diet with 5\% SBO to growing-finishing pigs support the present findings. A study [24] was conducted where growing finishing pigs were fed diets devoid of added fat or diets formulated with $5 \%$ beef tallow, poultry fat, or SBO. Fat source did not affect ADG, ADFI or G:F from that study [24]. A more recent study [25] where growingfinishing pigs were fed diets containing either $5 \%$ SBO or $5 \%$ CWG and a significant improved ADG response pigs fed SBO but not the CWG was observed. The ADFI and feed efficiency G:F from the present study (not shown in the table) were improved in both diets with added fat when compared with pigs fed the control diet containing no added fat. Previous work [26] reported growing-finishing pigs fed increasing CWG concentrations (0, $2.5 \%$, and $5 \%)$ had improved ADG $(P<0.05)$ and feed efficiency $(P<0.01)$ but had no effect on ADFI support the present findings in part. The present findings with high fat concentrations that require a substance Solka Floc $^{\circledR}$ to prevent ration ingredients from separating during the feeding process having no effect on pig performance are in agreement with previous findings $[27,28]$. Pigs fed $40 \%$ SBO diet with a low-density diluent, showed no response of compensatory ADFI. Cunningham et al.[29] reported that refined cellulose (Solka $\mathrm{Floc}^{\circledR}$ ) has a zero-energy value for growing pigs and that ADFI did not increase to compensate for cellulose in the diet. However, this work is in contrast to results $[30,31]$ when pigs fed $40 \%$ CWG and SBO diets had similar ADG and improved feed efficiency compared with pigs fed a control diet. Any performance differences associated with Solka Floc ${ }^{\circledR}$ could have been masked by including SBO in the diet.

The calculated average daily energy intake (ADEI) for the control group was similar to those for all treatment groups except for the $40 \%$ CWG group. Feeding CWG or SBO linearly $(P<0.001$, and $P<$ 0.05 , respectively) increased the lean gain efficiency, which almost paralleled that of feed efficiency. This result on lean efficiency agrees with previous data [32]. It is expected that inclusion of supplemental fat in diets fed to grow-finish swine will cause an increase in dietary energy density and result in decreased ADFI and increased feed efficiency with or without a change in ADG. Unfortunately, the authors are unable to calculate the efficiency of dietary energy being converted to energy of edible product.

Table 3: Growth and performance characteristics of finishing pigs fed diets containing choice white grease or soybean oil ${ }^{1}$.

\begin{tabular}{|c|c|c|c|c|c|c|c|c|c|c|c|c|}
\hline \multirow{3}{*}{ Item } & \multicolumn{12}{|c|}{ Treatments $^{2}$} \\
\hline & \multirow{2}{*}{ Control } & \multirow{2}{*}{$\begin{array}{l}10 \% \\
\text { CWG }\end{array}$} & \multirow{2}{*}{$\begin{array}{l}20 \% \\
\text { CWG }\end{array}$} & \multirow{2}{*}{$\begin{array}{l}30 \% \\
\text { CWG }\end{array}$} & \multirow{2}{*}{$\begin{array}{l}40 \% \\
\text { CWG }\end{array}$} & \multirow{2}{*}{$\begin{array}{l}10 \% \\
\text { SBO }\end{array}$} & \multirow{2}{*}{$\begin{array}{l}20 \% \\
\text { SBO }\end{array}$} & \multirow{2}{*}{$\begin{array}{l}30 \% \\
\text { SBO }\end{array}$} & \multirow{2}{*}{$\begin{array}{l}40 \% \\
\text { SBO }\end{array}$} & \multicolumn{3}{|c|}{ Contrasts $^{3}$} \\
\hline & & & & & & & & & & SEM $^{4}$ & CWG & SBO \\
\hline $\mathrm{ADFI}^{5}(\mathrm{~kg} / \mathrm{d})$ & $3.14^{\mathrm{a}}$ & $2.83^{\text {bcde }}$ & $2.96^{a b c}$ & $2.58^{\text {cde }}$ & $2.17^{f}$ & $3.01^{\mathrm{ab}}$ & $2.71^{\text {bcde }}$ & $2.48^{\text {def }}$ & $2.35^{\mathrm{ef}}$ & 0.144 & $* * *$ & $* * *$ \\
\hline $\operatorname{ADG}^{6}(\mathrm{~kg} / \mathrm{d})$ & 0.88 & 0.85 & 1.03 & 0.98 & 0.89 & 0.92 & 0.96 & 0.92 & 0.93 & 0.074 & & \\
\hline Feed efficiency $(\mathrm{g} / \mathrm{kg})$ & $276^{c}$ & $295^{c}$ & $347^{b}$ & $381^{\mathrm{ab}}$ & $403^{a}$ & $\left.11.9\right|^{b}$ & $11.3 \mathrm{~b}^{\mathrm{bc}}$ & $10.9 \mathrm{f}^{\mathrm{bc}}$ & $10.4^{\mathrm{bc}}$ & 0.574 & & \\
\hline$A D E I^{8}$ & $11.9^{\mathrm{ab}}$ & $11.3^{\mathrm{abc}}$ & $12.4^{\mathrm{a}}$ & $11.5^{\mathrm{abc}}$ & $10.2^{c}$ & $307^{c}$ & $354^{b}$ & $370^{\mathrm{ab}}$ & $394^{\mathrm{a}}$ & 134 & $* * *$ & $* * *$ \\
\hline Efficiency of lean gain ${ }^{9,10}(\mathrm{~g} / \mathrm{kg})$ & $97^{e}$ & $105^{\text {de }}$ & $113^{\text {cde }}$ & $131^{\mathrm{ab}}$ & $116^{\mathrm{a}}$ & $110^{\text {de }}$ & $120^{\mathrm{bcd}}$ & $127^{\mathrm{abc}}$ & $128^{\mathrm{ab}}$ & 6.15 & $* * *$ & $*$ \\
\hline
\end{tabular}

${ }^{1}$ Least squares means and contrasts.

${ }^{2} \mathrm{Abbr} .10 \%, 20 \%, 30 \%$, and $40 \%$ refer to amount of calories in diet from lipids, CWG = Choice white grease, SBO = Soybean oil.

${ }^{3}$ Contrast $=$ linear by amount of dietary lipid.

${ }^{4} n=6$ for all treatments, except for $40 \%$ CWG when $n=5$.

${ }^{5} \mathrm{ADFI}=$ average daily feed intake.

${ }^{6} \mathrm{ADG}=$ average daily gain.

${ }^{7}$ Feed efficiency $=\mathrm{g} B W$ gain $/ \mathrm{kg}$ feed intake.

${ }^{8} \mathrm{ADEl}$ Average daily energy intake $=\mathrm{ADFI} \times$ calculated metabolizable energy $(\mathrm{Mcal} / \mathrm{kg})$.

${ }^{9}$ Efficiency of lean gain $(\mathrm{g} / \mathrm{kg})=\mathrm{LDOT}$ (lean gain per day on test) $/$ ADFI.

${ }^{10}$ Used to calculate LDOT.

abcdef Means in the same row with a different superscript differ $(P<0.05), * P<0.05$, and $* * * P<0.001$ 


\section{Carcass characteristics}

Most carcass characteristics showed inconsistent individual treatment differences for each dietary lipid with no definite tendencies (Table 4). It is important to know how the fat treatments affected carcass traits in order to understand the economic benefit when high fat diets are fed to grow - finish pigs and is the reason for including results where no differences were observed. Pigs fed CWG tended, however, to have more 10th rib backfat than did those pigs fed SBO. Pigs fed the 20\% CWG, 30\% CWG, 40\% CWG, and 40\% SBO diets showed tendencies for increased 10th rib backfat, which is in contrast to previous work [32] that suggest that the increase resulted from decreased protein to energy dietary ratios. Backfat thickness data from this study partially agree with reports that backfat increases in pigs fed diets containing added fat [33-35]. Lean muscle produced per day on test seemed to be greater for pigs fed the fat-supplemented diets compared with those fed the control diet. Similarly, for the carcass composition, pigs fed the fat-supplemented diets tended to have more fat and skin and less lean muscle than did pigs fed the control diet. This last observation differs from previous findings [36] where no carcass lean content change was observed when pigs consumed a fat-supplemented diet that included peanuts. Similarly, the present backfat increase observed when high fat diets are fed differs with previous [24] where no carcass weight, dressing percentage, or first rib, last rib, or last lumbar vertebrae backfat differences from carcasses from pigs fed different fat sources. Similarly, no change in backfat or loin depth and lean percentage was observed when pigs were fed either CWG or SBO when compared with control diets with no added fat [25].

\section{Fatty acid composition of adipose tissue}

The changes in fatty acid compositions in 10th rib backfat were more pronounced for the pigs fed SBO than for the pigs fed CWG (Tables 5-7). This result was expected because CWG is derived primarily from porcine fat and thus is more similar in composition to backfat when compared to the fatty acid composition for SBO. Increasing CWG in grow-finish pig diets did not influence the linoleic or linolenic acid concentrations in the outer, middle, or inner 10th rib backfat layer. In general, the dietary CWG decreased stearic and palmitic acids but increased palmitoleic and oleic acids of 10th rib backfat. Overall, dietary CWG decreased SFA and increased monounsaturated fatty acids (MUFA) with no effect on polyunsaturated fatty acids (PUFA) in the outer, middle, and inner 10th rib backfat layers. In the middle and inner 10th rib backfat layers, the MUFA responses were linear $(P<0.001)$ with increasing dietary CWG, which contains a relatively high oleic acid proportion as well as MUFA (Table 2). Decreased SFA and increased MUFA for backfat have been reported when pigs were fed diets containing peanuts with similar MUFA concentrations as CWG [37].

Increasing dietary SBO fed to grow-finish pigs linearly $(\mathrm{P}<0.001)$ affected every fatty acid concentration from the three layers of

Table 4: Carcass characteristics and composition of finishing pigs fed diets containing choice white grease or soybean oil ${ }^{1}$.

\begin{tabular}{|c|c|c|c|c|c|c|c|c|c|c|}
\hline \multirow{2}{*}{ Item } & \multicolumn{10}{|c|}{ Treatments $^{2}$} \\
\hline & Control & $10 \%$ CWG & $20 \%$ CWG & $30 \%$ CWG & $40 \%$ CWG & $10 \%$ SBO & $20 \%$ SBO & $30 \%$ SBO & $40 \%$ SBO & SEM $^{34}$ \\
\hline Characteristic $^{5} 10$ th rib backfat, $\mathrm{cm}$ & 2.5 & 2.41 & 2.94 & 2.99 & 2.93 & 2.54 & 2.58 & 2.56 & 2.94 & 0.32 \\
\hline Lean color score ${ }^{6}$ & $2.83^{\mathrm{ab}}$ & $2.83 \mathrm{~h}^{\mathrm{ab}}$ & $3.00 a$ & $2.83^{\mathrm{ab}}$ & $2.35^{b}$ & $2.67^{\mathrm{ab}}$ & $2.83^{\mathrm{ab}}$ & $3.00^{\mathrm{a}}$ & $2.67^{\mathrm{ab}}$ & 0.17 \\
\hline Marbling score ${ }^{6}$ & $2.67^{\mathrm{ab}}$ & $2.67^{\mathrm{ab}}$ & $2.83 a$ & $2.50^{\mathrm{ab}}$ & $2.33^{\mathrm{ab}}$ & $2.67^{\mathrm{ab}}$ & $2.00^{\mathrm{b}}$ & $2.83^{\mathrm{a}}$ & $2.33^{\mathrm{ab}}$ & 0.27 \\
\hline Lean firmness score ${ }^{6}$ & $2.83^{d}$ & $2.50^{\mathrm{de}}$ & $2.83^{d}$ & $2.67^{\mathrm{de}}$ & $2.35^{\mathrm{de}}$ & $2.50^{\mathrm{de}}$ & $2.33^{\mathrm{de}}$ & $2.83^{d}$ & 2.17e & 0.27 \\
\hline Carcass weight, kg & $80.7^{\mathrm{a}}$ & $81.2^{\mathrm{ab}}$ & $83.9^{b c}$ & $85.2^{c}$ & $83.6^{b c}$ & $83.4^{\mathrm{bc}}$ & $83.4^{\mathrm{abc}}$ & $82.1^{\mathrm{ab}}$ & $82.3^{\mathrm{ab}}$ & 1 \\
\hline Carcass length, cm & 80.8 & 81.1 & 81.5 & 81.3 & 81.1 & 81.9 & 81.8 & 82.6 & 80.7 & 0.9 \\
\hline Dressing, \% & $74.2^{\mathrm{ab}}$ & $74.3^{\mathrm{ab}}$ & $74.9^{\mathrm{ab}}$ & $75.1^{\mathrm{a}}$ & $74.3^{\mathrm{ab}}$ & $74.8^{\mathrm{ab}}$ & $74.3^{\mathrm{ab}}$ & $73.6^{a}$ & $74.1^{\mathrm{ab}}$ & 0.5 \\
\hline LDOT7, g/d & 298 & 298 & 333 & 338 & 313 & 328 & 321 & 315 & 306 & 18 \\
\hline Longissimus muscle area, $\mathrm{cm}^{2}$ & 38.2 & 35.6 & 37.6 & 38 & 38.3 & 39.7 & 38.1 & 35.8 & 35 & 2.1 \\
\hline \multicolumn{11}{|l|}{ Composition } \\
\hline Lean, \% & 50.2 & 47.3 & 48 & 46 & 48.5 & 48.2 & 48.1 & 48.4 & 46.9 & 1.9 \\
\hline Fat, \% & $30.7^{a}$ & $32.9^{\mathrm{ab}}$ & $33.5^{\mathrm{ab}}$ & $36.6^{b}$ & $33.1^{\mathrm{ab}}$ & $33.7^{\mathrm{ab}}$ & $33.8^{\mathrm{ab}}$ & $31.6^{\mathrm{ab}}$ & $34.7^{\mathrm{ab}}$ & 2.1 \\
\hline Bone, \% & $13.8^{\mathrm{ab}}$ & $14.9^{a}$ & $13.9^{\mathrm{ab}}$ & $12.4^{\mathrm{a}}$ & $13.3^{\mathrm{ab}}$ & $13.0^{b}$ & $13.7^{\mathrm{ab}}$ & $14.7^{a}$ & $13.5^{\mathrm{ab}}$ & 0.6 \\
\hline Skin, \% & $5.33^{a}$ & $4.89^{\mathrm{ab}}$ & $4.67^{\mathrm{ab}}$ & $4.91^{\mathrm{ab}}$ & $5.18^{\mathrm{ab}}$ & $5.10^{\mathrm{ab}}$ & $4.50^{b}$ & $5.27^{\mathrm{a}}$ & $4.87^{\mathrm{ab}}$ & 0.26 \\
\hline
\end{tabular}

${ }^{1}$ Least squares means.

${ }^{2} \mathrm{Abbr} .10 \%, 20 \%, 30 \%$, and $40 \%$ refer to the dietary caloric coming from lipids, CWG = Choice white grease, SBO = Soybean oil.

${ }^{3}$ Standard error of the mean.

${ }^{4} n=6$ for all treatments, except for $40 \%$ CWG $n=5$.

${ }^{5} \mathrm{NPPC}, 1991$.

${ }^{6}$ Scores: 1 to 5 .

${ }^{7} \mathrm{LDOT}=$ Lean produced per day on test.

${ }^{a b c} M e a n s$ in the same row with a different superscript differ $(P<0.05)$.

${ }^{d e}$ Means in the same row with a different superscript differ $(P<0.10)$. 
Table 5: Fatty acid composition of the outer $10^{\text {th }}$ rib backfat layer from finishing pigs fed diets containing choice white grease or soybean oil ${ }^{1}$.

\begin{tabular}{|c|c|c|c|c|c|c|c|c|c|c|c|c|}
\hline \multicolumn{10}{|c|}{ Treatments ${ }^{2}$} & \multicolumn{3}{|c|}{ Contrasts $^{3}$} \\
\hline Item & Control & $10 \%$ CWG & $20 \%$ CWG & $30 \%$ CWG & $40 \%$ CWG & $10 \%$ SBO & $20 \%$ SBO & $30 \%$ SBO & $40 \%$ SBO & \multirow{2}{*}{ SEM $^{4}$} & \multirow{2}{*}{ CWG } & \multirow{2}{*}{ SBC } \\
\hline Fatty acids ${ }^{5}$ & & & & & & & & & & & & \\
\hline $14: 0$ & $1.29^{\mathrm{a}}$ & $1.23^{\mathrm{a}}$ & $1.29^{a}$ & $1.24^{\mathrm{a}}$ & $1.21^{\mathrm{a}}$ & $1.18^{\mathrm{a}}$ & $0.91^{b}$ & $0.78^{b c}$ & $0.65^{c}$ & 0.05 & & $* * *$ \\
\hline $16: 0$ & $26.8^{f}$ & $25.2^{\mathrm{fg}}$ & $24.5^{g}$ & $23.4^{\mathrm{g}}$ & $24.0^{\mathrm{g}}$ & $23.3^{\mathrm{g}}$ & $19.7^{\mathrm{g}}$ & $16.8^{i}$ & $15.0^{i}$ & 0.72 & & $* * *$ \\
\hline $16: 1$ & $2.39^{\mathrm{ac}}$ & $2.73^{\mathrm{abc}}$ & $3.02^{b}$ & $3.01^{b}$ & $3.10^{\mathrm{b}}$ & $2.16^{c}$ & $1.41^{d}$ & $1.26^{d}$ & $1.19^{d}$ & 0.13 & & $* * *$ \\
\hline $18: 0$ & $13.2^{f}$ & $11.1^{\mathrm{g}}$ & $9.9^{\mathrm{gh}}$ & $9.1^{h}$ & $10.2^{\mathrm{gh}}$ & $10.2^{\mathrm{gh}}$ & $9.5^{\mathrm{h}}$ & $7.4^{i}$ & $6.9^{i}$ & 0.56 & & $* * *$ \\
\hline $18: 1$ & $40.4^{f}$ & $44.2^{\mathrm{g}}$ & $46.7^{\mathrm{h}}$ & $47.5^{\mathrm{h}}$ & $45.5^{\mathrm{gh}}$ & $36.7^{i}$ & $32.0^{\mathrm{j}}$ & $30.1^{\mathrm{jk}}$ & $29.2^{\mathrm{k}}$ & 0.79 & & $* * *$ \\
\hline $18: 2$ & $14.1^{\mathrm{a}}$ & $13.8^{\mathrm{a}}$ & $13.0^{\mathrm{a}}$ & $13.9^{\mathrm{a}}$ & $14.0^{\mathrm{a}}$ & $23.5^{b}$ & $32.2^{\mathrm{c}}$ & $36.8^{d}$ & $41.3^{e}$ & 1.16 & & $* * *$ \\
\hline $18: 3$ & $0.39^{f}$ & $0.33^{f}$ & $0.32^{f}$ & $0.37^{f}$ & $0.38^{f}$ & $1.53^{\mathrm{g}}$ & $3.25^{h}$ & $3.76^{i}$ & $4.53^{j}$ & 0.18 & & $* * *$ \\
\hline Total SFA & $41.8^{f}$ & $37.9^{9}$ & $36.1^{\mathrm{gh}}$ & $34.3^{h}$ & $35.8^{\mathrm{gh}}$ & $35.0^{\mathrm{gh}}$ & $30.4^{i}$ & $25.4^{j}$ & $22.9^{j}$ & 1.24 & & $* * *$ \\
\hline Total MUFA & $43.4^{f}$ & $47.7^{\mathrm{g}}$ & $50.4^{h}$ & $51.3^{h}$ & $49.4^{\mathrm{gh}}$ & $39.5^{i}$ & $33.7^{\mathrm{j}}$ & $32.6^{\mathrm{jk}}$ & $30.7^{k}$ & 0.82 & & $* * *$ \\
\hline Total PUFA ${ }^{8}$ & $14.8^{f}$ & $14.4^{f}$ & $13.5^{j}$ & $14.5^{j}$ & $14.7^{j}$ & $25.5^{g}$ & $35.9^{h}$ & $42.0^{i}$ & $46.5^{j}$ & 1.29 & & $* * *$ \\
\hline$P: S$ & $0.36^{f}$ & $0.38^{f}$ & $0.38^{f}$ & $0.42^{f}$ & $0.41^{f}$ & $0.74^{\mathrm{g}}$ & $1.21^{\mathrm{h}}$ & $1.69^{i}$ & $2.13^{j}$ & 0.1 & & $* * *$ \\
\hline U:S & $1.41^{f}$ & $1.64^{f}$ & $1.78^{\mathrm{fg}}$ & $1.92^{\mathrm{gh}}$ & $1.81^{\mathrm{fg}}$ & $1.88^{\mathrm{g}}$ & $2.33^{h}$ & $2.99^{i}$ & $3.50^{\mathrm{j}}$ & 0.15 & & $* * *$ \\
\hline
\end{tabular}

${ }^{1}$ Least squares means and contrasts.

${ }^{2} \mathrm{Abbr}$. 10\%, 20\%, 30\%, and 40\% refer to amount of calories in diet from lipids, CWG = Choice white grease, SBO = Soybean oil.

${ }^{3}$ Contrast $=$ linear by amount of dietary lipid.

${ }^{4} n=6$ for all treatments, except for $40 \%$ CWG $n=5$.

${ }^{5}$ Fatty acids are reported as a weight percentage of total fatty acids identified, SFA = total saturated fatty acids, MUFA = total monounsaturated fatty acids, PUFA = total polyunsaturated fatty acids, P:S = ratio of total polyunsaturated to total saturated fatty acids, U:S = ratio of total unsaturated (mono- and poly-) to total saturated fatty acids.

${ }^{6}$ Also includes 15:0, 17:0, 20:0, and 22:0.

${ }^{7}$ Also includes $15: 1,17: 1,20: 1$, and $24: 1$

${ }^{8}$ Also includes 20:3, 20:4, and 22:2.

abcde Means in the same row with a different superscript differ $(P<0.01), * P<0.05, * * P<.001$, and $* * * P<0.001$

fghijk Means in the same row with a different superscript differ $(P<0.05)$.

Table 6: Fatty acid composition of middle $10^{\text {th }}$ rib backfat layer backfat from finishing pigs fed diets containing choice white grease or soybean oil ${ }^{1}$.

\begin{tabular}{|c|c|c|c|c|c|c|c|c|c|c|c|c|}
\hline \multicolumn{13}{|c|}{ Treatments ${ }^{2}$} \\
\hline Item & \multirow{2}{*}{ Control } & \multirow{2}{*}{$10 \%$ CWG } & \multirow{2}{*}{$20 \%$ CWG } & \multirow{2}{*}{$30 \%$ CWG } & \multirow{2}{*}{$40 \%$ CWG } & \multirow{2}{*}{$10 \%$ SBO } & \multirow{2}{*}{$20 \%$ SBO } & \multirow{2}{*}{$30 \%$ SBO } & \multirow{2}{*}{$40 \%$ SBO } & \multicolumn{3}{|c|}{ Contrasts $^{3}$} \\
\hline Fatty acids ${ }^{5}$ & & & & & & & & & & SEM $^{4}$ & CWG & SBO \\
\hline 14:0 & $1.32^{\mathrm{a}}$ & $1.21^{\mathrm{ab}}$ & $1.23^{\mathrm{ab}}$ & $1.17^{\mathrm{bc}}$ & $1.08^{c}$ & $1.15^{\mathrm{bc}}$ & $0.79^{d}$ & $0.67^{d}$ & $0.44^{e}$ & 0.044 & & $* * *$ \\
\hline 16:0 & $28.1^{a}$ & $26.3^{\mathrm{ab}}$ & $25.0^{\mathrm{bc}}$ & $23.8^{c}$ & $23.8^{\mathrm{c}}$ & $24.2^{c}$ & $19.1^{d}$ & $16.6^{e}$ & $13.2^{f}$ & 0.68 & $*$ & $* * *$ \\
\hline $16: 1$ & $1.94^{\mathrm{a}}$ & $2.16^{a}$ & $2.55^{b}$ & $2.61^{b}$ & $2.64^{b}$ & $1.15^{c}$ & $0.86^{d}$ & $0.73^{\mathrm{de}}$ & $0.55^{e}$ & 0.109 & $* *$ & $* * *$ \\
\hline 18:0 & $15.4^{\mathrm{a}}$ & $13.9^{\mathrm{ab}}$ & $11.8^{\mathrm{cd}}$ & $11.2^{\mathrm{cd}}$ & $11.3^{\mathrm{cd}}$ & $12.5^{b c}$ & $10.8^{d}$ & $8.6^{d}$ & $7.4^{e}$ & 0.58 & $* *$ & $* * *$ \\
\hline $18: 1$ & $39.0^{\mathrm{a}}$ & $42.5^{b}$ & $45.5^{c}$ & $46.3^{d}$ & $46.9^{d}$ & $34.5^{d}$ & $30.0^{e}$ & $28.7^{\mathrm{e}}$ & $26.6^{f}$ & 0.6 & $* * *$ & $* * *$ \\
\hline $18: 2$ & $12.9^{\mathrm{g}}$ & $12.5^{\mathrm{g}}$ & $12.5^{\mathrm{g}}$ & $12.7^{\mathrm{g}}$ & $12.9^{\mathrm{g}}$ & $23.5^{h}$ & $33.8^{i}$ & $39.8^{\mathrm{j}}$ & $44.9^{k}$ & 1.1 & & $* * *$ \\
\hline $18: 3$ & $0.30^{\mathrm{g}}$ & $0.25^{\mathrm{g}}$ & $0.28^{\mathrm{g}}$ & $0.32^{\mathrm{g}}$ & $0.32^{\mathrm{g}}$ & $1.46^{\mathrm{h}}$ & $3.22^{i}$ & $4.18^{\mathrm{j}}$ & $4.96^{k}$ & 0.14 & & $* * *$ \\
\hline Total SFA ${ }^{6}$ & $45.3^{\mathrm{a}}$ & $41.8^{b}$ & $38.5^{\mathrm{h}}$ & $36.6^{h}$ & $36.5^{\mathrm{h}}$ & $38.1^{\mathrm{h}}$ & $31.0^{i}$ & $26.0^{\mathrm{j}}$ & $21.3^{k}$ & 1.2 & $* *$ & $* * *$ \\
\hline Total MUFA ${ }^{7}$ & $41.5^{a}$ & $45.3^{b}$ & $48.7^{\mathrm{h}}$ & $50.0^{h}$ & $50.1^{h}$ & $36.5^{i}$ & $31.4^{j}$ & $29.5^{k}$ & $27.9^{k}$ & 0.6 & $* * *$ & $* * *$ \\
\hline Total PUFA ${ }^{8}$ & $13.3^{\mathrm{a}}$ & $12.9^{a}$ & $12.9^{\mathrm{a}}$ & $13.4^{\mathrm{a}}$ & $13.3^{a}$ & $25.3^{b}$ & $37.6^{h}$ & $44.4^{i}$ & $50.8^{j}$ & 1.2 & & $* * *$ \\
\hline$P: S$ & $0.30^{\mathrm{a}}$ & $0.31^{\mathrm{a}}$ & $0.34^{\mathrm{a}}$ & $0.37^{a}$ & $0.37^{a}$ & $0.68^{b}$ & $1.26^{h}$ & $1.74^{i}$ & $2.41^{\mathrm{j}}$ & 0.08 & & $* * *$ \\
\hline U:S & $1.22^{\mathrm{a}}$ & $1.40^{\mathrm{ab}}$ & $1.61^{\text {bh }}$ & $1.74^{\mathrm{h}}$ & $1.74^{h}$ & $1.64^{\mathrm{bh}}$ & $2.28^{i}$ & $2.89^{j}$ & $3.72^{k}$ & 0.12 & * & $* * *$ \\
\hline
\end{tabular}

${ }^{1}$ Least squares means and contrasts.

${ }^{2} \mathrm{Abbr} .10 \%, 20 \%, 30 \%$, and $40 \%$ refer to amount of calories in diet from lipids, CWG = Choice white grease, SBO = Soybean oil

${ }^{3}$ Contrast $=$ linear by amount of dietary lipid.

${ }^{4} n=6$ for all treatments, except for $40 \%$ CWG $n=5$.

${ }^{5}$ Fatty acids are reported as a weight percentage of total fatty acids, SFA = total saturated fatty acids, MUFA = total monounsaturated fatty acids. PUFA = total polyunsaturated fatty acids, $\mathrm{P}: \mathrm{S}=$ ratio of total polyunsaturated to total saturated fatty acids, U:S = ratio of total unsaturated (monoand poly-) to total saturated fatty acids.

${ }^{6}$ Also includes 15:0, 17:0, and 20:0.

${ }^{7}$ Also includes $17: 1,20: 1,22: 1$, and $24: 1$.

${ }^{8}$ Also includes 20:2, 20:3, and 20:4.

abcdef Means in the same row with a different superscript differ $(P<0.05)$.

ghijk Means in the same row with a different superscript differ $(P<0.01),{ }^{*} P<0.05,{ }^{*} P<.001$, and $* * * P<0.001$. 
Table 7: Fatty acid composition of inner $10^{\text {th }}$ rib backfat layer from finishing pigs fed diets containing choice white grease or soybean oil ${ }^{1}$.

\begin{tabular}{|c|c|c|c|c|c|c|c|c|c|c|c|c|}
\hline \multicolumn{13}{|c|}{ Treatments² } \\
\hline Item & & \multirow{2}{*}{$10 \%$ CWG } & \multirow{2}{*}{$20 \%$ CWG } & \multirow{2}{*}{$30 \%$ CWG } & \multirow{2}{*}{$40 \%$ CWG } & \multirow{2}{*}{$10 \%$ SBO } & \multirow{2}{*}{$20 \%$ SBO } & \multirow{2}{*}{$30 \%$ SBO } & \multirow{2}{*}{$40 \%$ SBO } & \multicolumn{3}{|c|}{ Contrasts $^{3}$} \\
\hline Fatty acids ${ }^{5}$ & Control & & & & & & & & & SEM $^{4}$ & CWG & SBO \\
\hline $14: 0$ & $1.39^{\mathrm{a}}$ & $1.20^{\mathrm{b}}$ & $1.21^{\mathrm{b}}$ & $1.17^{b}$ & $1.17^{b}$ & $1.13^{b}$ & $0.80^{c}$ & $0.68^{d}$ & $0.42^{e}$ & 0.048 & & $* * *$ \\
\hline $16: 0$ & $29.0^{\mathrm{a}}$ & $26.2^{b}$ & $25.9^{\mathrm{b}}$ & $23.9^{c}$ & $24.7^{b c}$ & $24.0^{c}$ & $19.2^{d}$ & $16.5^{\mathrm{e}}$ & $13.2^{f}$ & 0.7 & & $* * *$ \\
\hline $16: 1$ & $2.32^{a}$ & $2.20^{\mathrm{a}}$ & $2.26^{\mathrm{a}}$ & $2.58^{a}$ & $2.65^{a}$ & $1.52^{\mathrm{b}}$ & $0.90^{c}$ & $0.70^{c}$ & $0.53^{c}$ & 0.17 & $*$ & $* * *$ \\
\hline $18: 0$ & $14.6^{a}$ & $13.0^{\mathrm{ab}}$ & $11.8^{b c}$ & $10.9^{c}$ & $11.3^{\mathrm{bc}}$ & $12.2^{\mathrm{bc}}$ & $10.7^{c}$ & $8.8^{\mathrm{d}}$ & $7.2^{\mathrm{d}}$ & 0.6 & $*$ & $* * *$ \\
\hline $18: 1$ & $40.1^{a}$ & $41.3^{a}$ & $44.7^{c}$ & $47.1^{c}$ & $45.5^{c}$ & $35.3^{c}$ & $30.0^{d}$ & $28.2^{\mathrm{d}}$ & $26.9^{d}$ & 1.2 & $*$ & $* * *$ \\
\hline $18: 2$ & $11.5^{\mathrm{g}}$ & $14.6^{\mathrm{g}}$ & $12.9^{\mathrm{g}}$ & $13.1^{\mathrm{g}}$ & $13.3^{\mathrm{g}}$ & $23.7^{\mathrm{h}}$ & $34.3^{i}$ & $40.3^{j}$ & $46.5^{k}$ & 1.3 & & $* * *$ \\
\hline $18: 3$ & $0.27^{g}$ & $0.47^{g}$ & $0.27^{\mathrm{g}}$ & $0.31^{\mathrm{g}}$ & $0.33^{g}$ & $1.35^{\mathrm{h}}$ & $3.22^{i}$ & $4.03^{j}$ & $4.77^{\mathrm{k}}$ & 0.16 & & $* * *$ \\
\hline Total SFA & $45.4^{a}$ & $40.8^{b}$ & $39.3^{b c}$ & $36.3^{c}$ & $37.6^{\mathrm{bc}}$ & $37.6^{\mathrm{bc}}$ & $31.0^{d}$ & $26.2^{\mathrm{e}}$ & $21.0^{f}$ & 1.3 & & $* * *$ \\
\hline Total MUFA ${ }^{7}$ & $42.8^{a}$ & $44.0^{\mathrm{a}}$ & $47.4^{b}$ & $50.1^{b}$ & $48.7^{b}$ & $37.2^{c}$ & $31.1^{d}$ & $29.0^{d}$ & $27.5^{e}$ & 1.1 & $* * *$ & $* * *$ \\
\hline Total PUFA ${ }^{8}$ & $11.8^{\mathrm{g}}$ & $15.2^{\mathrm{g}}$ & $13.3^{\mathrm{g}}$ & $13.5^{g}$ & $13.8^{\mathrm{g}}$ & $25.2^{h}$ & $37.8^{i}$ & $44.8^{j}$ & $51.6^{k}$ & 1.4 & & $* * *$ \\
\hline$P: S$ & $0.26^{g}$ & $0.38^{g}$ & $0.34^{\mathrm{g}}$ & $0.38^{g}$ & $0.37^{g}$ & $0.69^{h}$ & $1.27^{i}$ & $1.73^{j}$ & $2.48^{k}$ & 0.077 & & $* * *$ \\
\hline U:S & $1.21^{\mathrm{a}}$ & $1.47^{\mathrm{ab}}$ & $1.57^{b}$ & $1.76^{b}$ & $1.67^{b}$ & $1.68^{b}$ & $2.28^{c}$ & $2.84^{d}$ & $3.80^{\mathrm{e}}$ & 0.11 & & $* * *$ \\
\hline
\end{tabular}

${ }^{1}$ Least squares means and contrasts.

${ }^{2} \mathrm{Abbr}$. $10 \%, 20 \%, 30 \%$, and $40 \%$ refer to amount of calories in diet from lipids, CWG $=$ Choice white grease, SBO = Soybean oil.

${ }^{3}$ Contrast $=$ linear by amount of dietary lipid.

${ }^{4} n=6$ for all treatments, except for $40 \%$ CWG $n=5$.

${ }^{5}$ Fatty acids are reported as a weight percentage of total fatty acids, SFA = total saturated fatty acids, MUFA = total monounsaturated fatty acids, PUFA = total polyunsaturated fatty acids, P:S = ratio of total polyunsaturated to total saturated fatty acids, U:S = ratio of total unsaturated (monoand poly-) to total saturated fatty acids.

${ }^{6}$ Also includes 15:0, 17:0, and 20:0.

${ }^{7}$ Also includes 17:1, and 20:1.

${ }^{8}$ Also includes 20:2, 20:3, and 20:4.

abcdef Means in the same row with a different superscript differ $(P<0.05)$.

ghijk Means in the same row with a different superscript differ $(P<0.01), * P<0.05, * * P<.001$, and $* * * P<0.001$.

10th rib backfat. The overall effect of including $40 \%$ of energy from SBO in the diet was that backfat SFA decreased by almost one-half, MUFA decreased by almost one third, and PUFA increased more than two-fold in the outer 10th rib backfat layer compared with the 10th rib backfat from pigs fed the control diet. The PUFA to SFA (P:S) ratio increased by almost five-fold, and the unsaturated fatty acid to SFA (U:S) ratio increased by almost $150 \%$ for the outer 10 th rib backfat layer from pigs fed the $40 \%$ SBO diet compared with the backfat from pigs fed the control diet.

Soybean oil is rich in PUFA, with linoleic acid being the dominant fatty acid. In all three 10th rib backfat layers from the pigs fed SBO, PUFA increased as MUFA and SFA decreased. Additionally, all three 10th rib backfat layers increased in linolenic acid. A similar increased linoleic acid response in backfat has been reported when pigs were fed diets containing increased linoleic acid content [38-41]. All linolenic acid in the three 10th rib backfat layers from pigs fed all dietary treatments was alphalinolenic acid, which was in the experimental diets. Romans et al. [42] showed a similar increased alpha-linolenic acid response in backfat when pigs were fed diets containing ground flaxseed. These results are consistent with the previous results [43] where grow-finish pigs fed the $5 \%$ SBO as an added fat dietary treatment had increased PUFA in subcutaneous fat. In that same study, the innermost backfat layer had the greatest SFA concentration and the lowest MUFA concentration when compared with SFA in the middle and outer backfat layers.
The relatively high oleic acid concentration in backfat from pigs fed the control diet agrees with previous work $[44,45]$ that reported that oleic acid tends to be found in high concentrations in the adipose tissue from pigs fed fat-free diets. The control diet could be considered a very low-fat diet because it was calculated to contain $7 \%$ of energy as lipid.

The basic fatty acid profile change resulting from dietary treatment with CWG or SBO was repeated in all three 10th backfat layers. This similarity in changes among the three 10th backfat layers that results from feeding supplemental fat agrees with the changes observed previously reported [46]. Tenth-rib backfat fatty acid data from this study agree with reports that fatty acids profiles from subcutaneous adipose tissue generally reflect the fatty acid composition from the dietary fat source [41,47-49].

The perirenal adipose tissue fatty acid profiles (Table 8) and an intermuscular fat deposit within the ham (Table 9) showed similar changes to dietary lipids as did the three 10th rib backfat layers. Previous data agree with the report that perirenal adipose tissue decreases in SFA and MUFA and increases in PUFA for pigs fed roasted soybeans compared with pigs fed a control diet [50]. For all dietary treatments, perirenal adipose tissue contained more SFA, less MUFA $(P<0.05)$, especially oleic acid, and similar PUFA content when compared with the 10th rib backfat. These findings agree with previous findings $[12,51]$. The inter-muscular fat deposit within the ham revealed a fatty acid profile similar to that from the outer 10th rib backfat layer for all dietary 
Table 8: Fatty acid composition of perirenal adipose tissue from finishing pigs fed diets containing choice white grease or soybean oil ${ }^{1}$.

\begin{tabular}{|c|c|c|c|c|c|c|c|c|c|c|c|c|}
\hline \multicolumn{13}{|c|}{ Treatments ${ }^{2}$} \\
\hline Item & \multirow{2}{*}{ Control } & \multirow{2}{*}{$10 \%$ CWG } & \multirow{2}{*}{$20 \%$ CWG } & \multirow{2}{*}{$30 \%$ CWG } & \multirow{2}{*}{$40 \%$ CWG } & \multirow{2}{*}{$10 \%$ SBO } & \multirow{2}{*}{$20 \%$ SBO } & \multirow{2}{*}{$30 \%$ SBO } & \multirow{2}{*}{$40 \%$ SBO } & \multicolumn{3}{|c|}{ Contrasts $^{3}$} \\
\hline Fatty acids ${ }^{5}$ & & & & & & & & & & SEM $^{4}$ & CWG & SBO \\
\hline $14: 0$ & $1.47^{\mathrm{a}}$ & $1.35^{\mathrm{a}}$ & $1.43^{\mathrm{a}}$ & $1.46^{\mathrm{a}}$ & $1.31^{a}$ & $1.33^{\mathrm{a}}$ & $1.00^{b}$ & $0.85^{b}$ & $0.60^{c}$ & 0.061 & & $* * *$ \\
\hline $16: 0$ & $30.4^{f}$ & $28.9^{\mathrm{fg}}$ & $27.3^{\mathrm{gh}}$ & $27.0^{\mathrm{gh}}$ & $26.1^{h}$ & $26.4^{\mathrm{h}}$ & $21.8^{i}$ & $18.8^{\mathrm{j}}$ & $15.3^{\mathrm{k}}$ & 0.81 & $*$ & $* * *$ \\
\hline $16: 1$ & $1.77^{f}$ & $2.03^{f}$ & $2.36^{g}$ & $2.61^{\mathrm{g}}$ & $2.45^{\mathrm{g}}$ & $1.22^{\mathrm{h}}$ & $0.79^{i}$ & $0.62^{\mathrm{ij}}$ & $0.47^{j}$ & 0.12 & $* *$ & $* * *$ \\
\hline 18:0 & $17.6^{f}$ & $15.8^{\mathrm{fg}}$ & $14.1^{\mathrm{gh}}$ & $13.2^{h}$ & $13.0^{\mathrm{h}}$ & $15.7^{\mathrm{fg}}$ & $13.6^{\mathrm{h}}$ & $10.4^{i}$ & $8.7^{i}$ & 0.73 & $*$ & $* * *$ \\
\hline $18: 1$ & $35.1^{f}$ & $39.8^{\mathrm{g}}$ & $40.7^{\mathrm{g}}$ & $43.0^{\mathrm{g}}$ & $30.2^{\mathrm{g}}$ & $30.2^{h}$ & $26.7^{\mathrm{hi}}$ & $26.0^{i}$ & $24.5^{i}$ & 1.26 & & $* *$ \\
\hline $18: 2$ & $12.2^{\mathrm{a}}$ & $10.7^{a}$ & $12.6^{a}$ & $13.0^{\mathrm{a}}$ & $12.7^{\mathrm{a}}$ & $22.7^{b}$ & $32.3^{c}$ & $38.9^{d}$ & $45.1^{\mathrm{e}}$ & 1.54 & & $* * *$ \\
\hline $18: 3$ & $0.30^{f}$ & $0.21^{f}$ & $0.33^{f}$ & $0.33^{f}$ & $1.54^{f}$ & $1.54^{\mathrm{g}}$ & $2.96^{h}$ & $3.64^{i}$ & $4.75^{j}$ & 0.19 & & $* * *$ \\
\hline Total SFA & $50.1^{f}$ & $46.6^{\mathrm{fg}}$ & $43.4^{\mathrm{gh}}$ & $42.2^{h}$ & $41.0^{\mathrm{hi}}$ & $43.9^{\mathrm{gh}}$ & $36.8^{i}$ & $30.3^{j}$ & $24.8^{k}$ & 1.49 & $*$ & $* * *$ \\
\hline Total MUFA & $37.3^{f}$ & $42.3^{g}$ & $43.6^{\mathrm{gh}}$ & $44.2^{\mathrm{gh}}$ & $45.9^{h}$ & $31.7^{i}$ & $27.7^{\mathrm{j}}$ & $26.8^{j}$ & $25.0^{\mathrm{j}}$ & 1.24 & & $* * *$ \\
\hline Total PUFA ${ }^{8}$ & $12.6^{a}$ & $11.1^{\mathrm{a}}$ & $13.0^{\mathrm{a}}$ & $13.5^{a}$ & $13.2^{\mathrm{a}}$ & $24.4^{\mathrm{b}}$ & $35.5^{c}$ & $42.9^{d}$ & $50.2^{\mathrm{e}}$ & 1.7 & & $* * *$ \\
\hline$P: S$ & $0.25^{f}$ & $0.24^{f}$ & $0.30^{f}$ & $0.32^{f}$ & $0.58^{f}$ & $0.58^{g}$ & $1.00^{\mathrm{h}}$ & $1.48^{i}$ & $2.07^{j}$ & 0.09 & & $* * *$ \\
\hline U:S & $1.0^{f}$ & $1.1^{\mathrm{fg}}$ & $1.3^{\mathrm{fg}}$ & $1.4^{\mathrm{g}}$ & $1.4^{\mathrm{gh}}$ & $1.3^{\mathrm{fg}}$ & $1.8^{\mathrm{h}}$ & $2.4^{i}$ & $3.1^{\mathrm{j}}$ & 0.12 & & $* * *$ \\
\hline
\end{tabular}

${ }^{1}$ Least squares means and contrasts.

${ }^{2} \mathrm{Abbr} .10 \%, 20 \%, 30 \%$, and $40 \%$ refer to amount of calories in diet from lipids, CWG = Choice white grease, SBO = Soybean oil.

${ }^{3}$ Contrast $=$ linear by amount of dietary lipid.

${ }^{4} n=6$ for all treatments, except for $40 \%$ CWG $n=5$.

${ }^{5}$ Fatty acids are reported as a weight percentage of total fatty acids, SFA = total saturated fatty acids, MUFA = total monounsaturated fatty acids, PUFA $=$ total polyunsaturated fatty acids, $\mathrm{P}: \mathrm{S}=$ ratio of total polyunsaturated to total saturated fatty acids, $\mathrm{U}: \mathrm{S}=$ ratio of total unsaturated (monoand poly-) to total saturated fatty acids.

${ }^{6}$ Also includes 15:0, 17:0, and 20:0.

${ }^{7}$ Also includes 15:1, 17:1, 20:1, 22:1, and 24:1.

${ }^{8}$ Also includes 20:2, 20:3, 20:4, 22:2, and 22:6.

abcde Means in the same row with a different superscript differ $(P<0.01), * * P<0.01$, and $* * * P<0.001$.

fghijk Means in the same row with a different superscript differ $(P<0.05)$.

Table 9: Fatty acid composition of an inter-muscular fat deposit within the ham from finishing pigs fed diets containing choice white grease or soybean oil'.

\begin{tabular}{|c|c|c|c|c|c|c|c|c|c|c|c|c|}
\hline \multicolumn{13}{|c|}{ Treatments $^{2}$} \\
\hline Item & \multirow{2}{*}{ Control } & \multirow{2}{*}{$10 \%$ CWG } & \multirow{2}{*}{$20 \%$ CWG } & \multirow{2}{*}{$30 \%$ CWG } & \multirow{2}{*}{$40 \%$ CWG } & \multirow{2}{*}{$10 \%$ SBO } & \multirow{2}{*}{$20 \%$ SBO } & \multirow{2}{*}{$30 \%$ SBO } & \multirow{2}{*}{$40 \%$ SBO } & \multicolumn{3}{|c|}{ Contrasts $^{3}$} \\
\hline Fatty acids ${ }^{5}$ & & & & & & & & & & SEM $^{4}$ & CWG & SBO \\
\hline $14: 0$ & $1.37^{\mathrm{a}}$ & $1.28^{\mathrm{a}}$ & $1.44^{\mathrm{a}}$ & $1.35^{\mathrm{a}}$ & $1.25^{\mathrm{a}}$ & $1.28^{a}$ & $1.07^{b}$ & $0.81^{c}$ & $0.67^{c}$ & 0.07 & & $* * *$ \\
\hline $16: 0$ & $26.7^{\mathrm{a}}$ & $25.7^{\mathrm{a}}$ & $26.6^{a}$ & $25.1^{\mathrm{a}}$ & $24.5^{\mathrm{ab}}$ & $25.8^{a}$ & $21.9^{b}$ & $18.2^{c}$ & $16.4^{c}$ & 0.9 & & $* * *$ \\
\hline $16: 1$ & $2.88^{\mathrm{ab}}$ & $2.97^{\mathrm{ab}}$ & $3.51^{c}$ & $3.19^{\mathrm{ac}}$ & $2.84^{\mathrm{ab}}$ & $2.56^{b}$ & $1.93^{d}$ & $1.23^{\mathrm{e}}$ & $1.46^{\text {de }}$ & 0.19 & & $* * *$ \\
\hline 18:0 & $11.4^{\mathrm{a}}$ & $10.6^{\mathrm{ab}}$ & $10.0^{\mathrm{ab}}$ & $9.3^{b c}$ & $10.1^{\mathrm{ab}}$ & $10.2^{\mathrm{ab}}$ & $9.6^{\mathrm{b}}$ & $7.7^{c d}$ & $7.2^{\mathrm{d}}$ & 0.6 & & $* * *$ \\
\hline $18: 1$ & $41.7^{\mathrm{a}}$ & $43.8^{a}$ & $41.3^{a}$ & $43.6^{a}$ & $44.8^{\mathrm{a}}$ & $35.7^{b}$ & $31.1^{b c}$ & $30.4^{c}$ & $29.9^{c}$ & 1.8 & & $*$ \\
\hline $18: 2$ & $13.8^{f}$ & $14.1^{f}$ & $15.1^{f}$ & $15.3^{f}$ & $14.6^{f}$ & $21.9^{g}$ & $30.9^{h}$ & $37.7^{i}$ & $39.4^{i}$ & 1.3 & & $* * *$ \\
\hline $18: 3$ & $0.29^{a}$ & $0.29^{a}$ & $0.39^{a}$ & $0.43^{\mathrm{a}}$ & $0.39^{a}$ & $1.07^{b}$ & $2.07^{c}$ & $2.93^{d}$ & $3.39^{e}$ & 0.16 & & $* * *$ \\
\hline Total SFA ${ }^{6}$ & $39.9^{a}$ & $38.0^{\mathrm{a}}$ & $38.6^{a}$ & $36.3^{\mathrm{ab}}$ & $36.3^{\mathrm{ab}}$ & $37.9^{a}$ & $32.9^{b}$ & $27.1^{\mathrm{c}}$ & $24.6^{c}$ & 1.5 & & $* * *$ \\
\hline Total MUFA ${ }^{7}$ & $45.6^{a}$ & $47.4^{\mathrm{a}}$ & $45.6^{\mathrm{a}}$ & $47.6^{a}$ & $48.4^{\mathrm{a}}$ & $38.8^{b}$ & $33.7^{c}$ & $32.2^{\mathrm{c}}$ & $31.9^{c}$ & 1.7 & & $* *$ \\
\hline Total PUFA ${ }^{8}$ & $14.5^{f}$ & $14.6^{f}$ & $15.8^{f}$ & $16.1^{f}$ & $15.2^{f}$ & $23.4^{\mathrm{g}}$ & $33.5^{\mathrm{h}}$ & $40.7^{i}$ & $43.5^{i}$ & 1.5 & & $* * *$ \\
\hline $\mathrm{P}: \mathrm{S}$ & $0.37^{a}$ & $0.39^{a b}$ & $0.41^{\mathrm{ab}}$ & $0.45^{\mathrm{ab}}$ & $0.42^{\mathrm{ab}}$ & $0.63^{b}$ & $1.07^{c}$ & $1.55^{d}$ & $1.83^{e}$ & 0.1 & & $* * *$ \\
\hline U:S & $1.52^{\mathrm{a}}$ & $1.65^{a}$ & $1.61^{\mathrm{a}}$ & $1.77^{\mathrm{ab}}$ & $1.78^{\mathrm{ab}}$ & $1.68^{a}$ & $2.12^{b}$ & $2.76^{c}$ & $3.15^{c}$ & 0.15 & & $* * *$ \\
\hline
\end{tabular}

${ }^{1}$ Least squares means and contrasts.

${ }^{2} \mathrm{Abbr}$. 10\%, 20\%, 30\%, and 40\% refer to amount of calories in diet from lipids, CWG = Choice white grease, SBO = Soybean oil.

${ }^{3}$ Contrast $=$ linear by amount of dietary lipid.

${ }^{4} n=6$ for all treatments, except for $40 \%$ CWG $n=5$.

${ }^{5}$ Fatty acids are reported as a weight percentage of total fatty acids, SFA = total saturated fatty acids, MUFA = total monounsaturated fatty acids, PUFA = total polyunsaturated fatty acids, $\mathrm{P}: \mathrm{S}=$ ratio of total polyunsaturated to total saturated fatty acids, $\mathrm{U}: \mathrm{S}=$ ratio of total unsaturated (monoand poly-) to total saturated fatty acids.

${ }^{6}$ Also includes 15:0, 17:0, 20:0, and 22:0.

${ }^{7}$ Also includes $17: 1,20: 1,22: 1$, and 24:1.

${ }^{8}$ Also includes 20:2, 20:3, 20:4, 22:2, and 22:6.

abced Means in the same row with a different superscript differ $(P<.05)$.

fghi Means in the same row with a different superscript differ $(P<.01), * P<.05, * * P<.01$ and $* * * P<0.001$. 
treatments. The slight, but insignificant, differences for these two internal adipose tissues compared with that for subcutaneous adipose tissues are probably results from anatomical location and unknown unique physiological functions.

\section{Fatty acid composition of skeletal muscles}

Adding CWG as an energy source in the diets fed to grow-finish pigs had no to minimal effects on proportions of individual fatty acids, content of SFA and MUFA, P:S and U:S, and lipid content from the longissimus muscle (Table 10). Seerley et al. [52] reported a decrease in oleic acid and an increase in linoleic acid in longissimus muscle from pigs fed a diet that contained poultry fat. Pigs fed CWG showed similar tendencies as did the pigs fed poultry fat, which contains similar amounts of MUFA as CWG but is more unsaturated than CWG. When SBO was included as an energy source in the diets fed to grow-finish pigs, however, SFA and MUFA decreased as the dietary SBO increased. Polyunsaturated fatty acids increased linearly $(P<0.001)$ by more than $140 \%$ in the longissimus muscle from pigs as dietary SBO increased from lowest to highest concentrations. This response agrees with work when pigs were fed full-fat soybeans [53]. The longissimus muscle lipid content from pigs tended to decrease as dietary SBO increased.

Fatty acid compositions from the triceps brachii muscle (Table
11) and from the biceps femoris muscle (Table 12) revealed similar responses to dietary treatments as did the longissimus muscle fatty acid composition. With few exceptions, the contents of SFA, MUFA, and PUFA from the triceps brachii muscle and biceps femoris muscle were similar for all dietary treatment groups including the control group. The exceptions were with the SBO-fed pigs where PUFA content in both muscles increased with increasing dietary SBO.

The longissimus muscle, however, contained slightly greater SFA and MUFA content and slightly less PUFA content than did either the triceps brachii muscle or the biceps femoris muscle. Concentration of myristic, palmitic, and linolenic acids in lipids from longissimus muscle from pigs were not affected $(P>0.05)$ by dietary CWG. This lack of response agrees with the findings reported by Jurgens (Table 10) [46]. The PUFA concentrations in lipids from the longissimus muscle were less $(P<0.05$, except $40 \%$ SBO treatment group $P<0.06)$ than those in lipids from the triceps brachii muscle for all dietary treatment groups. Further, the PUFA from the longissimus muscle were less $(P<$ $0.05)$ than those from the biceps femoris muscle for all dietary SBO treatments. The MUFA from the longissimus muscle were greater $(P<0.05$, except $30 \%$ CWG treatment group $P<0.07)$ than those of the triceps brachii muscle for all dietary CWG treatment groups and the control treatment group.

Table 10: Fatty acid composition, and lipid and dry matter content of longissimus muscle from finishing pigs fed diets containing choice white grease or soybean oil ${ }^{1}$.

\begin{tabular}{|c|c|c|c|c|c|c|c|c|c|c|c|c|}
\hline \multicolumn{13}{|c|}{ Treatments $^{2}$} \\
\hline Item & \multirow{2}{*}{ Control } & \multirow{2}{*}{$10 \%$ CWG } & \multirow{2}{*}{$20 \%$ CWG } & \multirow{2}{*}{$30 \%$ CWG } & \multirow{2}{*}{$40 \%$ CWG } & \multirow{2}{*}{$10 \%$ SBO } & \multirow{2}{*}{$20 \%$ SBO } & \multirow{2}{*}{$30 \%$ SBO } & \multirow{2}{*}{$40 \%$ SBO } & \multicolumn{3}{|c|}{ Contrasts $^{3}$} \\
\hline Fatty acids ${ }^{5}$ & & & & & & & & & & SEM $^{4}$ & CWG & SBC \\
\hline $14: 0$ & $1.33^{\mathrm{ab}}$ & $1.28^{\mathrm{abc}}$ & $1.32^{\mathrm{ab}}$ & $1.37^{\mathrm{a}}$ & $1.36^{\mathrm{a}}$ & $1.37^{\mathrm{a}}$ & $1.19^{b c}$ & $1.14^{c}$ & $0.97^{d}$ & 0.055 & & $* * *$ \\
\hline $16: 0$ & $27.5^{\mathrm{a}}$ & $26.9^{a}$ & $26.4^{\mathrm{a}}$ & $26.0^{\mathrm{ab}}$ & $26.1^{\mathrm{ab}}$ & $26.8^{a}$ & $24.5^{b}$ & $22.3^{c}$ & $19.3^{d}$ & 0.65 & & $* * *$ \\
\hline $16: 1$ & & $4.10^{\mathrm{a}}$ & $3.98^{\mathrm{ab}}$ & $3.80^{\mathrm{ab}}$ & $3.50^{\mathrm{b}}$ & $3.81^{\mathrm{ab}}$ & $2.87^{c}$ & $2.83^{d}$ & $1.94^{d}$ & 0.18 & & $* * *$ \\
\hline 18:0 & $12.28^{a}$ & $12.00^{\mathrm{ab}}$ & $11.19^{a b}$ & $11.01^{\mathrm{bc}}$ & $11.24^{\mathrm{ab}}$ & $11.74^{\mathrm{ab}}$ & $11.48^{\mathrm{ab}}$ & $10.07^{c}$ & $8.73^{d}$ & 0.4 & & $* * *$ \\
\hline $18: 1$ & $43.2^{\mathrm{ab}}$ & $46.0^{\mathrm{a}}$ & $45.2^{\mathrm{a}}$ & $44.4^{\mathrm{ab}}$ & $40.8^{\mathrm{bc}}$ & $39.1^{c}$ & $38.5^{c}$ & $32.5^{d}$ & $31.6^{\mathrm{e}}$ & 1.37 & $*$ & $* * *$ \\
\hline $18: 2$ & $7.4^{\mathrm{a}}$ & $6.8^{\mathrm{a}}$ & $8.1^{\mathrm{a}}$ & $9.9^{a b}$ & $12.8^{\mathrm{b}}$ & $12.6^{b}$ & $16.6^{c}$ & $25.8^{d}$ & $31.4^{e}$ & 1.24 & $* *$ & $* * *$ \\
\hline $18: 3$ & $0.19^{f}$ & $0.19^{f}$ & $0.33^{f}$ & $0.25^{f}$ & $0.54^{\mathrm{fg}}$ & $0.60^{\mathrm{fg}}$ & $1.03^{g}$ & $2.41^{\mathrm{h}}$ & $3.16^{i}$ & 0.18 & & $* * *$ \\
\hline Total SFA ${ }^{6}$ & $41.4^{\mathrm{a}}$ & $40.4^{\mathrm{ab}}$ & $39.2^{\mathrm{abc}}$ & $38.6^{\mathrm{bc}}$ & $39.0^{a b c}$ & $40.2^{\mathrm{abc}}$ & $37.4^{\mathrm{c}}$ & $33.8^{d}$ & $29.3^{e}$ & 1.03 & & $* * *$ \\
\hline Total MUFA ${ }^{7}$ & $49.8^{\mathrm{ab}}$ & $51.7^{\mathrm{a}}$ & $51.0^{\mathrm{a}}$ & $50.2^{a}$ & $46.4^{b}$ & $45.0^{c}$ & $43.5^{c}$ & $36.1^{d}$ & $34.6^{d}$ & 1.26 & $*$ & $* * *$ \\
\hline Total PUFA ${ }^{8}$ & $8.8^{\mathrm{a}}$ & $8.0^{\mathrm{a}}$ & $9.8^{a}$ & $11.2^{\mathrm{ab}}$ & $14.6^{b}$ & $14.8^{b}$ & $19.1^{c}$ & $30.0^{d}$ & $36.1^{e}$ & 1.45 & $* *$ & $* * *$ \\
\hline$P: S$ & $0.21^{\mathrm{ab}}$ & $0.20^{\mathrm{a}}$ & $0.25^{\mathrm{ab}}$ & $0.29^{\mathrm{ab}}$ & $0.38^{\mathrm{bc}}$ & $0.38^{\mathrm{bc}}$ & $0.52^{c}$ & $0.90^{d}$ & $1.26^{\mathrm{e}}$ & 0.059 & & $* * *$ \\
\hline U:S & $1.42^{\mathrm{a}}$ & $1.48^{\mathrm{ab}}$ & $1.57^{\mathrm{ab}}$ & $1.60^{\mathrm{ab}}$ & $1.58^{\mathrm{ab}}$ & $1.50^{\mathrm{ab}}$ & $1.68^{b}$ & $1.97^{c}$ & $2.44^{d}$ & 0.079 & & $* * *$ \\
\hline Total lipids, \% & $3.24^{\mathrm{ab}}$ & $3.82^{\mathrm{a}}$ & $3.25^{\mathrm{ab}}$ & $3.47^{a}$ & $3.18^{\mathrm{ab}}$ & $2.47^{b}$ & $2.60^{b}$ & $3.07^{a b}$ & $3.46^{a}$ & 0.28 & $*$ & $*$ \\
\hline $\mathrm{DM}^{9}, \%$ & 26.92 & 27.01 & 26.42 & 26.8 & 27.43 & 26.25 & 26.14 & 26.55 & 26.68 & 0.32 & & \\
\hline
\end{tabular}

${ }^{1}$ Least squares means and contrasts.

${ }^{2} \mathrm{Abbr}$. $10 \%, 20 \%, 30 \%$, and $40 \%$ refer to amount of calories in diet from lipids, CWG = Choice white grease, SBO = Soybean oil.

${ }^{3}$ Contrast $=$ linear by amount of dietary lipid.

${ }^{4} n=6$ for all treatments, except for $40 \%$ CWG $n=5$.

${ }^{5}$ Fatty acids are reported as a weight percentage of total fatty acids, SFA = total saturated fatty acids, MUFA = total monounsaturated fatty acids, PUFA $=$ total polyunsaturated fatty acids, $\mathrm{P}: \mathrm{S}=$ ratio of total polyunsaturated to total saturated fatty acids, $\mathrm{U}: \mathrm{S}=$ ratio of total unsaturated (monoand poly-) to total saturated fatty acids.

${ }^{6}$ Also includes 15:0, 17:0, 20:0, and 22:0.

${ }^{7}$ Also includes $15: 1,17: 1,20: 1$, and 22:1.

${ }^{8}$ Also includes 20:3, 20:4, 22:2, and 22:6.

${ }^{9} \mathrm{DM}=$ Dry matter.

abcde Means in the same row with a different superscript differ $(P<0.05)$.

fghi Means in the same row with a different superscript differ $(P<0.01), * P<0.05$, and ${ }^{* * *} P<0.001$. 
Table 11: Fatty acid composition of triceps brachii muscle from finishing pigs fed diets containing choice white grease or soybean oil ${ }^{1}$.

\begin{tabular}{|c|c|c|c|c|c|c|c|c|c|c|c|c|}
\hline \multicolumn{13}{|c|}{ Treatments ${ }^{2}$} \\
\hline Item & & \multirow{2}{*}{$10 \%$ CWG } & \multirow{2}{*}{$20 \%$ CWG } & \multirow{2}{*}{$30 \%$ CWG } & \multirow{2}{*}{$40 \%$ CWG } & \multirow{2}{*}{$10 \% \mathrm{SBO}$} & \multirow{2}{*}{$20 \%$ SBO } & \multirow{2}{*}{$30 \%$ SBO } & \multirow{2}{*}{$40 \%$ SBO } & \multicolumn{3}{|c|}{ Contrasts $^{3}$} \\
\hline Fatty acids ${ }^{5}$ & Control & & & & & & & & & SEM $^{4}$ & CWG & SBO \\
\hline $14: 0$ & $1.06^{\mathrm{ab}}$ & $1.03^{\mathrm{ab}}$ & $1.08^{\mathrm{ab}}$ & $1.06^{\mathrm{ab}}$ & $0.94^{\mathrm{abc}}$ & $1.10^{\mathrm{a}}$ & $0.90^{b c}$ & $0.78^{c}$ & $0.52^{d}$ & 0.064 & & $* * *$ \\
\hline $16: 0$ & $24.7^{f}$ & $23.9^{f}$ & $23.8^{f}$ & $23.3^{f}$ & $24.3^{f}$ & $23.2^{f}$ & $20.5^{g}$ & $19.2^{\mathrm{gh}}$ & $17.6^{h}$ & 0.63 & & $* * *$ \\
\hline $16: 1$ & $3.36^{\mathrm{a}}$ & $3.44^{\mathrm{a}}$ & $3.52^{\mathrm{a}}$ & $3.24^{\mathrm{ab}}$ & $2.87^{b}$ & $3.08^{\mathrm{ab}}$ & $2.04^{c}$ & $1.57^{d}$ & $1.21^{\mathrm{d}}$ & 0.17 & $*$ & $* * *$ \\
\hline $18: 0$ & $11.2^{\mathrm{a}}$ & $10.8^{a}$ & $10.5^{a}$ & $10.3^{\mathrm{ab}}$ & $10.7^{a}$ & $10.7^{a}$ & $10.4^{\mathrm{ab}}$ & $9.5^{b c}$ & $9.0^{c}$ & 0.36 & & $* * *$ \\
\hline $18: 1$ & $37.3^{\mathrm{abc}}$ & $41.8^{a}$ & $41.6^{a}$ & $41.3^{\mathrm{ab}}$ & $35.0^{\text {cd }}$ & $36.9^{b c}$ & $31.9^{\text {de }}$ & $29.5^{e}$ & $28.9^{e}$ & 1.7 & $*$ & $* * *$ \\
\hline $18: 2$ & $14.1^{\mathrm{abc}}$ & $12.1^{\mathrm{b}}$ & $13.5^{b c}$ & $14.8^{\mathrm{abc}}$ & $18.0^{\mathrm{ac}}$ & $18.1^{\mathrm{a}}$ & $26.4^{d}$ & $32.1^{\mathrm{e}}$ & $33.2^{\mathrm{e}}$ & 1.6 & $*$ & $* * *$ \\
\hline $18: 3$ & $0.44^{\mathrm{a}}$ & $0.28^{a}$ & $0.32^{\mathrm{a}}$ & $0.38^{\mathrm{a}}$ & $0.49^{a}$ & $0.99^{\mathrm{a}}$ & $1.96^{b}$ & $3.15^{c}$ & $3.71^{c}$ & 0.28 & & $* * *$ \\
\hline Total SFA ${ }^{6}$ & $37.5^{\mathrm{a}}$ & $36.2^{\mathrm{a}}$ & $35.8^{a}$ & $35.0^{\mathrm{a}}$ & $36.4^{\mathrm{a}}$ & $35.5^{a}$ & $32.3^{b}$ & $29.9^{b c}$ & $27.4^{c}$ & 0.9 & & $* * *$ \\
\hline Total MUFA ${ }^{7}$ & $44.1^{\mathrm{ab}}$ & $47.5^{a}$ & $47.4^{\mathrm{a}}$ & $46.8^{a}$ & $41.1^{b}$ & $42.1^{b}$ & $35.8^{c}$ & $32.7^{c}$ & $31.9^{c}$ & 1.7 & $*$ & $* * *$ \\
\hline Total PUFA ${ }^{8}$ & $18.4^{\mathrm{ab}}$ & $16.3^{a}$ & $16.9^{a}$ & $18.2^{\mathrm{ab}}$ & $22.5^{b}$ & $22.4^{b}$ & $31.9^{c}$ & $37.5^{d}$ & $40.7^{e}$ & 1.8 & $*$ & $* * *$ \\
\hline $\mathrm{P}: \mathrm{S}$ & $0.49^{a}$ & $0.45^{a}$ & $0.47^{a}$ & $0.52^{\mathrm{a}}$ & $0.62^{a}$ & $0.64^{a}$ & $1.00^{b}$ & $1.28^{c}$ & $1.52^{d}$ & 0.075 & & $* * *$ \\
\hline U:S & $1.67^{a}$ & $1.76^{\mathrm{a}}$ & $1.80^{\mathrm{a}}$ & $1.86^{\mathrm{a}}$ & $1.77^{\mathrm{a}}$ & $1.84^{\mathrm{a}}$ & $2.12^{b}$ & $2.37^{c}$ & $2.68^{d}$ & 0.089 & & $* * *$ \\
\hline
\end{tabular}

${ }^{1}$ Least squares means and contrasts.

${ }^{2} \mathrm{Abbr}$. 10\%, 20\%, 30\%, and 40\% refer to amount of calories in diet from lipids, CWG = Choice white grease, SBO = Soybean oil.

${ }^{3}$ Contrast $=$ linear by amount of dietary lipid, CWG vs SBO = interaction.

${ }^{4} n=6$ for all treatments, except for $40 \%$ CWG $n=5$.

${ }^{5}$ Fatty acids are reported as a weight percentage of total fatty acids, SFA = total saturated fatty acids, MUFA = total monounsaturated fatty acids, PUFA = total polyunsaturated fatty acids, $\mathrm{P}: \mathrm{S}=$ ratio of total polyunsaturated to total saturated fatty acids, U:S = ratio of total unsaturated (monoand poly-) to total saturated fatty acids.

${ }^{6}$ Also includes 15:0, 17:0, 20:0, and 22:0.

${ }^{7}$ Also includes 15:1, 17:1, 20:1, 22:1, and 24:1.

${ }^{8}$ Also includes 20:2, 20:3, 20:4, 22:2, and 22:6.

abcde Means in the same row with a different superscript differ $(P<0.05)$.

${ }^{\text {fah }}$ Means in the same row with a different superscript differ $(P<0.01), * P<0.05, * * P<0.01$, and $* * * P<0.001$.

Table 12: Fatty acid composition of biceps femoris muscle from finishing pigs fed diets containing choice white grease or soybean oil ${ }^{1}$.

\begin{tabular}{|c|c|c|c|c|c|c|c|c|c|c|c|c|}
\hline \multicolumn{13}{|c|}{ Treatments $^{2}$} \\
\hline Item & \multirow{2}{*}{ Control } & \multirow{2}{*}{$10 \%$ CWG } & \multirow{2}{*}{$20 \%$ CWG } & \multirow{2}{*}{$30 \%$ CWG } & \multirow{2}{*}{$40 \%$ CWG } & \multirow{2}{*}{$10 \%$ SBO } & \multirow{2}{*}{$20 \%$ SBO } & \multirow{2}{*}{$30 \%$ SBO } & \multirow{2}{*}{$40 \%$ SBO } & \multicolumn{3}{|c|}{ Contrasts $^{3}$} \\
\hline Fatty acids ${ }^{5}$ & & & & & & & & & & SEM $^{4}$ & CWG & SBO \\
\hline $14: 0$ & $1.10^{\mathrm{ab}}$ & $1.23^{\mathrm{a}}$ & $1.20^{\mathrm{ab}}$ & $1.04^{b}$ & $1.15^{\mathrm{ab}}$ & $1.23^{\mathrm{a}}$ & $1.03^{b}$ & $0.84^{c}$ & $.74^{c}$ & 0.068 & & $* * *$ \\
\hline $16: 0$ & $25.0^{\mathrm{ab}}$ & $25.4^{\mathrm{a}}$ & $24.6^{\mathrm{ab}}$ & $23.6^{\mathrm{b}}$ & $24.4^{\mathrm{ab}}$ & $24.6^{\mathrm{ab}}$ & $21.8^{c}$ & $19.9^{d}$ & $17.9^{d}$ & 0.61 & & $* * *$ \\
\hline $16: 1$ & $3.70^{\mathrm{ab}}$ & $3.79^{a}$ & $3.85^{\mathrm{a}}$ & $3.24^{b}$ & $3.24^{b}$ & $3.51^{\mathrm{ab}}$ & $2.51^{c}$ & $1.72^{d}$ & $1.60^{\mathrm{e}}$ & 0.18 & $*$ & $* * *$ \\
\hline 18:0 & $10.6^{\mathrm{ab}}$ & $10.8^{a}$ & $9.6^{\text {bcde }}$ & $9.1^{\text {de }}$ & $9.8^{\mathrm{abcd}}$ & $10.2^{\mathrm{abc}}$ & $9.3^{\text {cde }}$ & $8.6^{\mathrm{ef}}$ & $7.7^{f}$ & 0.38 & & $* * *$ \\
\hline $18: 1$ & $39.8^{\mathrm{ac}}$ & $44.4^{b}$ & $43.0^{\mathrm{ab}}$ & $42.0^{\mathrm{ab}}$ & $40.5^{a b c}$ & $37.3^{\text {cd }}$ & $34.9^{d}$ & $28.9^{\mathrm{e}}$ & $28.4^{e}$ & 1.7 & & $* * *$ \\
\hline $18: 2$ & $13.0^{\mathrm{ab}}$ & $10.4^{\mathrm{a}}$ & $12.7^{\mathrm{ab}}$ & $14.7^{\mathrm{bc}}$ & $14.5^{\mathrm{bc}}$ & $17.6^{c}$ & $24.5^{d}$ & $32.8^{e}$ & $36.3^{f}$ & 1.2 & $*$ & $* * *$ \\
\hline $18: 3$ & $.28^{\mathrm{a}}$ & $.16^{a}$ & $.23^{\mathrm{a}}$ & $.32^{\mathrm{a}}$ & $.29^{a}$ & $.68^{\mathrm{b}}$ & $1.74^{c}$ & $2.69^{d}$ & $3.41^{\mathrm{e}}$ & 0.12 & & $* * *$ \\
\hline Total SFA & $37.0^{\mathrm{a}}$ & $37.7^{\mathrm{a}}$ & $35.7^{\mathrm{ab}}$ & $34.1^{b c}$ & $35.8^{\mathrm{ab}}$ & $36.4^{\mathrm{ab}}$ & $32.4^{c}$ & $29.6^{d}$ & $26.6^{e}$ & 1 & & $* * *$ \\
\hline Total MUFA & $47.5^{a}$ & $50.4^{a}$ & $50.0^{\mathrm{a}}$ & $48.9^{a}$ & $47.0^{\mathrm{ab}}$ & $43.6^{b}$ & $39.5^{c}$ & $33.2^{\mathrm{d}}$ & $32.0^{d}$ & 1.4 & & $* * *$ \\
\hline Total PUFA ${ }^{8}$ & $15.5^{\mathrm{ab}}$ & $11.9^{a}$ & $14.3^{\mathrm{ab}}$ & $17.0^{\mathrm{bc}}$ & $17.1^{b c}$ & $19.9^{c}$ & $28.1^{d}$ & $37.2^{\mathrm{e}}$ & $41.3^{f}$ & 1.5 & $*$ & $* * *$ \\
\hline$P: S$ & $.42^{\mathrm{ab}}$ & $.32^{\mathrm{a}}$ & $.40^{\mathrm{ab}}$ & $.50^{\mathrm{b}}$ & $.47^{a b}$ & $.56^{\mathrm{b}}$ & $.88^{c}$ & $1.27^{d}$ & $1.58^{\mathrm{e}}$ & 0.064 & & $* * *$ \\
\hline U:S & $1.71^{\mathrm{ab}}$ & $1.65^{a}$ & $1.82^{\mathrm{ab}}$ & $1.93^{\mathrm{bc}}$ & $1.80^{\mathrm{ab}}$ & $1.77^{\mathrm{ab}}$ & $2.11^{c}$ & $2.40^{d}$ & $2.78^{e}$ & 0.082 & & $* * *$ \\
\hline
\end{tabular}

${ }^{1}$ Least squares means and contrasts.

${ }^{2} \mathrm{Abbr} .10 \%, 20 \%, 30 \%$, and $40 \%$ refer to amount of calories in diet from lipids, CWG = Choice white grease, SBO = Soybean oil

${ }^{3}$ Contrast $=$ linear by amount of dietary lipid.

${ }^{4} n=6$ for all treatments, except for $40 \%$ CWG $n=5$.

${ }^{5}$ Fatty acids are reported as a weight percentage of total fatty acids, SFA = total saturated fatty acids, MUFA = total monounsaturated fatty acids, PUFA = total polyunsaturated fatty acids, $\mathrm{P}: \mathrm{S}=$ ratio of total polyunsaturated to total saturated fatty acids, $\mathrm{U}: \mathrm{S}=$ ratio of total unsaturated (monoand poly-) to total saturated fatty acids.

${ }^{6}$ Also includes 15:0, 17:0, 20:0, and 22:0.

${ }^{7}$ Also includes 15:1, 17:1, 20:1, 22:1, and 24:1.

${ }^{8}$ Also includes 20:3, 20:4, 22:2, and 22:6.

abcdef Means in the same row with a different superscript differ $(P<0.05), * P<0.05, * * P<0.01$, and $* * * P<0.001$. 
Results from this experiment where grow-finish pigs were fed diets containing CWG and smaller SBO concentrations agree with previous work $[46,54]$ in that the major SFA was palmitic acid and the principal unsaturated fatty acid was oleic acid within porcine muscle and adipose tissues. When greater SBO concentrations, however, were incorporated into the diet, linoleic acid in muscle and adipose tissues became predominant. Fatty acid profiles for backfat, longissimus muscle, and perirenal adipose tissue from pigs fed control and SBO diets are consistent when similar diets are used [55]. In agreement with previous work [45,56-58], the present findings show linoleic acid concentrations within adipose and skeletal muscle tissues are highly dependent on the dietary linoleic acid amount. Linoleic acid was preferentially incorporated into adipose tissue at a higher rate than in skeletal muscle when pigs were fed diets with increased linoleic acid content. These results agree with the findings reported by Seerley et al. [59] who fed cooked soybeans to growing pigs.

Results from the present study contrast with previous work [60] where PUFA content from all adipose and skeletal muscle tissues examined changed only slightly when pigs were supplemented with whole peanuts, despite the high linoleic acid content found in peanut lipids. Longissimus muscle PUFA tended to be lesser for pigs fed CWG and were lesser for pigs fed the control diet $(P<0.02)$ and for pigs fed SBO diets $(P<0.001)$ than PUFA from the outer 10th rib backfat layer. This result is opposite of those reported by Rhee and co-workers [61]. The present findings agree with previous work $[62,63]$ who noted that SFA are preferentially deposited in perirenal rather than in subcutaneous adipose tissue and in the inner subcutaneous layer rather than the outer subcutaneous layer of adipose tissue. The preference pattern for unsaturated fatty acids is the opposite. The magnitude of the changes in skeletal muscle lipid composition resulting from supplemental dietary lipids was noticeably less than those from adipose tissue. Moreover, the fatty acid profile from dietary lipids was not always clearly exhibited in skeletal muscle lipids.

The practical aspects of feeding diets containing choice white grease (CWG) or soybean oil (SBO) at $10,20,30$, or $40 \%$ of total calories must be considered. These diets may not be practical for several reasons including 1 . Difficulty feeding - diets containing this much fat do not flow well and may bridge in the storage bins and / or feeders potentially causing out of feed events for

\section{References}

1 Stahly TF, Cromwell GL (1979) Effect of environmental temperature and dietary fat supplementation on the performance and carcass characteristics of growing and finishing swine. J Anim Sci 49: 14781488.

2 Keys A (1984) Serum cholesterol response to dietary cholesterol. Am J Clin Nutr 40: 351-359.

3 Rudel LL, Parks JS, Johnson FL, Babiak J (1986) Low density lipoproteins in atherosclerosis. J Lipid Res 27: 465-474.

4 Oliver MF (1987) Dietary fat and coronary heart disease. Br Heart J 58: 423-428. the pigs, 2. When high quantities of SBO (unsaturated fats) are included in the pigs' ration, the carcasses from these pigs will likely poor processing characteristics due to the soft fat, 3 . Soft bellies result from carcasses where pigs were fed diets high in unsaturated fats. The soft bellies are undesirable from the processor's perspective because they are difficult to slice and are objectionable to consumers, and 4 . Diets high in unsaturated fats have oxidative stability issues and may require supplemental dietary vitamin $\mathrm{E}$ to improve the fats stability.

In recent years, periodically fat has become relatively expensive because of worldwide energy demands. This economic change has increased the costs of adding fat to swine diets from SBO, CWG, or any other source and often prohibits including fat in growing-finishing diets because the expense exceeds the returns for its inclusion. Nutritionists and pork producers must continually evaluate the cost and returns for adding fat before it is routinely included in diets fed to growing-finishing pig.

\section{Conclusions and Implications}

The results from this study indicated that SBO can be included in a grow-finish diet for swine for the last $10 \mathrm{wk}$ of the finishing period to modify the fatty acid composition and tended to decrease the lipid content of pork without adversely affecting animal growth or meat characteristics. A follow up study was conducted where humans consumed the pork and lard from control and $40 \%$ SBO-fed pigs [64]. Consuming the redesigned pork foods (high PUFA content) resulted in a decrease in total plasma and LDL-cholesterol and shifted the fatty acid composition from SFAs to PUFAs in plasma and erythrocytes in the humans. Therefore, modifying the fatty acid composition of pork by dietary measures is a useful approach to improving the plasma lipid profile, especially LDL-cholesterol, of human consumers. No apparent advantage or disadvantage was obtained by including CWG in a swine finishing diet except for the fact that CWG can be included as an alternate and economical energy source when no other changes are desired.

\section{Acknowledgements}

This project was funded by the USDA-supported Center for Designing Foods to Improve Nutrition at lowa State University (Center no longer exists).

5 Grundy SM, Bilheimer D, Blackburn H, Brown WV, Kwiterovich PO, et al. (1982) AHA Committee Report: Rationale of the diet-heart statement of the American Heart Association. Report of Nutrition Committee. Nutrition Today 17: 16-20.

6 Nestel PJ (1987) Polyunsaturated fatty acids (n-3, n-6). Am J Clin Nutr 45: 1161-1167.

7 O'Connor LE, Kim JE, Campbell WW (2017) Total red meat intake of $\geq$ 0.5 servings/d does not negatively influence cardiovascular disease risk factors: a systematically searched meta-analysis of randomized controlled trials. Am J Clin Nutr 105: 57-69.

8 Siri-Tarino PW, Sun Q, Hu FB, Krauss R (2010) Meta-analysis of 
prospective cohort studies evaluating the association of saturated fat with cardiovascular disease. Am J Clin Nutr 91: 535-546.

9 Lawrence GD (2013) Dietary fats and health: Dietary recommendations in the context of scientific evidence. Adv Nutr 4: 294:302.

10 Skelley GC, Borgman RF, Handlin DL, Acton JC, McConnell JC, et al. (1975) Influence of diet on quality, fatty acids, and acceptability of pork. J Anim Sci 41: 1298-1304.

11 Wood JD (1984) Fat deposition and the quality of fat tissue in meat animals. Fats in Animal Nutrition. 41: 407-435.

12 Miller MF, Shackelford SD, Hayden KD, Reagan JO (1990) Determination of the alteration in fatty acid profiles, sensory characteristics, and carcass traits of swine fed elevated levels of monounsaturated fats in the diet. J Anim Sci 68: 1624-1631.

13 Selke E, Rohwedder WK, Dutton HJ (1980). Volatile components from trilinolein heated in air. J Am Oil Chem Soc 57: 75-80.

14 Selke E, Rohwedder WK, Dutton HJ (1977) Volatile components from trilinolein heated in air. J Am Oil Chem Soc 54: 62-67.

15 King-Brink M, Sebranek JG, (1993) Combustion method for determination of crude protein in meat and meat products: Collaborative study. J AOAC Internat 76: 787-793.

16 NRC (1988). Nutrient requirements of swine (9th Ed). National Academy Press. Washington, DC.

17 National Pork Producers Council (NPPC) (1991) Procedures to evaluate mark hog performance (3rd Ed). NPPC, Des Moines, lowa. 1-16.

18 Bligh EG, Dyer WJ (1959) A rapid method of total lipid extraction and purification. Can J Biochem Physiol 37: 911-917.

19 Lepage G, Roy C (1986) Direct trans esterification of all classes of lipids in a one-step reaction. J Lipid Res 27: 114-120.

20 SAS (1988). SAS User's Guide: Statistics. SAS Inst Inc Cary, NC.

21 Dove CR, Haydon KD (1992) The effect of copper and fat addition to the diets of weanling swine on growth performance and serum fatty acids. J Anim Sci 70: 805-810.

22 Myer RO, Combs GE (1991) Fat supplementation of diets containing a high level of oats for growing-finishing swine. J Anim Sci 69: 46654669.

23 Morgan CA, Noble RC, Cocchi M, McCartney R (1992) Manipulation of the fatty acid composition of pig meat lipids by dietary means. $J$ Sci Food Agric 58: 357-368.

24 Apple JK, Maxwell CV, Galloway DL, Hutchison S, Hamilton CR, et al. (2009) Interactive effects of dietary fat source and slaughter weight in growing-finishing swine: I. Growth performance and longissimus muscle fatty acid composition. J Anim Sci 87: 1407-1422.

25 Benz JM, Tokach MD, Dritz SS, Nelssen JL, DeRouchey JM, et al. (2011) Effects of choice white grease and soybean oil on growth performance, carcass characteristics, and carcass fat quality of growing-finishing pigs. J Anim Sci 89: 404-413.

26 Barnes KM, Winslow NR, Shelton AG, Hlusko KC, Azain MJ (2012) Effect of dietary conjugated linoleic acid on marbling and intramuscular adipocytes in pork. J Anim Sci 90: 1142-1149.

27 Jensen AH, Becker DE, Terrill SW (1959) Growth inhibitory effect of oat hulls in rations for growing-finishing swine. J Anim Sci 18: 13561361.

28 Hoefer JA, Pearson AM, Stevenson JW, Luecke RW (1963) Effect of fibrous feedstuffs fed during the finishing period of gain, feed efficiency and carcass characteristics of swine. Quart Bull Mich Agron Expt Sta 45: 480-483.

29 Cunningham HM, Friend DW, Nicholson JWG (1961) The effect of a purified source of cellulose on the growth and body composition of growing pigs. Can J Anim Sci 41: 120-125.

30 Teague HS, Hanson LE (1954) The effect of feeding different levels of a cellulosic material to swine. J Anim Sci 13: 206-214.

31 Baker DH, Becker DE, Jensen AH, Harmon BG (1968) Effect of dietary dilution on performance of finishing swine. J Anim Sci 26: 1332-1335.

32 Prince TJ (1987) Effects of dietary fat addition and nutrient density on performance and carcass composition of swine. J Anim Sci 65(Suppl 1): 301.

33 Allee GL, Romsos DR, Leveille GA, Baker DH (1972) Lipogenesis and enzymatic activity in pig adipose tissue as influenced by source of dietary fat. J Anim Sci 35: 41-47.

34 Leibbrandt VD, Hays VW, Ewan RC, Speer VC (1975) Effect of fat on performance of baby and growing pigs. J Anim Sci 40: 1081-1085.

35 Mersmann HJ (1984) Effect of sex on lipogenic activity in swine adipose tissue. J Anim Sci 58: 600-604.

36 West RL, Myer RO (1987) Carcass and meat quality characteristics and backfat fatty acid composition of swine as affected by the consumption of peanuts remaining in the field after harvest. J Anim Sci 65: 475-480.

37 Myer RO, Johnson DD, Knauft DA, Gorbet DW, Brendemuhl JH, et al. (1992) Effect of feeding high-oleic-acid peanuts to growing-finishing swine on resulting carcass fatty acid profile and on carcass and meat quality characteristics. J Anim Sci 70: 3734-3741.

38 Nordstrom JW, Behrends BR, Meade RJ, Thompson EH (1972) Effects of feeding high oil corns to growing-finishing swine. J Anim Sci 35 357-361.

39 Wood JD, Buxton PJ, Whittington FM, Enser M (1986) The chemical composition of fat tissues in the pig: effects of castration and feeding treatment. Livest Prod Sci 15: 73-82.

40 Adams KL, Jensen AH (1987) High-fat maize in diets for pigs and sows. Anim Feed Sci Tech17: 201-212.

41 Kellner TA, Prusa KJ, Patience JF (2014) Impact of dietary fat source and concentration and daily fatty acid intake on the composition of carcass fat and iodine value sampled in three regions of the pork carcass. J Anim Sci 99: 5485-5495.

42 Romans JR, Johnson RC, Wulf DM, Libal GW, Costello WJ (1995) Effects of ground flaxseed in swine diets on pig performance and on physical and sensory characteristics and omega-3 fatty acid content of pork: 1. Dietary level of flaxseed. J Anim Sci 73: 1982-1986.

43 Apple JK, Maxwell CV, Galloway DL, Hamilton CR, Yancey JWS (2009) Interactive effects of dietary fat source and slaughter weight in growing-finishing swine: II. Fatty acid composition of subcutaneous fat. J Anim Sci 87: 1423-1440.

44 Leat WMF, Cuthbertson A, Howard AN, Gresham GA (1964) Studies on pigs reared on semi-synthetic diets containing no fat, beef tallow and maize oil: composition of carcass and fatty acid composition of various depot fats. J Agric Sci 63: 311-317.

45 Kass ML, Pond WG, Walker Jr EF (1975) Significance of the synthesis of essential fatty acids in swine. J Anim Sci 41: 804-808.

46 Jurgens MH, Peo Jr EO, Vipperman PE, Mandigo RW (1970) Influence 
of dietary supplements of vitamin $\mathrm{D}_{3}$ and various fats on cholesterol and fatty acid composition of the blood and body of growingfinishing swine. J Anim Sci 30: 904-910.

47 Koch DE, Pearson AM, Magee WT, Hoefer JA, Schweigert BS (1968) Effect of diet on the fatty acid composition of pork fat. J Anim Sci 27 360-365.

48 Bowland JP (1972) Unprocessed rapeseed treated with propionic acid in diets of growing pigs: Performance, energy and protein digestibility, and nitrogen retention, carcass measurement, and fatty acid composition of backfat. Can J Anim Sci 52: 553-562.

49 Castell AG, Falk L (1980) Effects of dietary canola seed on pig performance and backfat composition. Can J Anim Sci 60: 795-797.

50 Villegas FJ, Hedrick HB, Veum TL, McFate KL, Thomas WK, et al. (1973) Effect of breed and diet containing cooked soybeans vs. extracted soybean meal on pork carcass characteristics. J Anim Sci 37: 443-449.

51 Busboom JR, Rule DC, Colin D, Heald T, Mazhar A (1991) Growth, carcass characteristics, and lipid composition of adipose tissue and muscle of pigs fed canola. J Anim Sci 69: 1101-1108.

52 Seerley RW, Briscoe JP, McCampbell HC (1978) A comparison of poultry and animal fat on performance, body composition and tissue lipids of swine. J Anim Sci 46: 1018-1023.

53 Leszczynski DE, Pikul J, Easter RA, McKeith FK, McLaren DG, et al. (1992) Characterization of lipid in loin and bacon from finishing pigs fed full-fat soybeans or tallow. J Anim Sci 70: 2175-2181.

54 Hilditch TP (1956) The chemical constitution of natural fats. John Wiley and Sons Inc, New York, $3^{\text {rd }}$ ed.

55 Christensen KD (1963) Various fatty acids in fat tissues of pigs of the Danish Landrace fed with coconut fat or soybean oil. Acta Agric Scand 13: 249-258.

56 Ellis NR, Isbell HS (1926) Soft pork studies II. The influence of the character of the ration upon the composition of the body fat of hogs. J Biol Chem 69: 219-238.

57 Ellis, NR, Hankins OG (1925) Soft pork studies I. Formation of fat in the pig on a ration moderately low in fat. J Biol Chem 66: 101-122.

58 Ellis NR, Zeller JH (1930) Soft pork studies. IV. The influence of a ration low in fat upon the composition of the body fat of hogs. J Biol Chem 89: 185-197.

59 Seerley RW, Emberson JW, McCampbell HC, Burdick D, Grimes LW (1974) Cooked soybeans in swine and rat diets. J Anim Sci 39: 18021091.

60 Chung RA, Lin CC (1965) Fatty acid content of pork cuts and variety meats as affected by different dietary lipids. J Food Sci 30: 860-864.

61 Rhee KS, Davidson TL, Knabe DA, Cross HR, Ziprin YA, et al. (1988) Effect of dietary high-oleic sunflower oil on pork carcass traits and fatty acid profiles of raw tissues. Meat Sci 24: 249-260.

62 Sink JD, Watkins JL, Ziegler JH, Miller RC (1964) Analysis of fat deposition in swine by gas-liquid chromatography. J Anim Sci 23: 121-125.

63 Lefaucheur L, Le Dividich J, Mourot J, Monin G, Ecolan P, et al. (1991) Influence of environmental temperature on growth, muscle and adipose tissue metabolism, and meat quality in swine J Anim Sci 69: 2844-2854.

64 Stewart JW, Kaplan ML, Beitz DC (2001) Pork with a high content of polyunsaturated fatty acids lowers LDL cholesterol in women. Am J Clin Nutr 74: 179-187. 